\title{
ANALYSE PREUSSISCHER SCHULBÜCHER ALS ZUGANG ZUM THEMA „SCHULBILDUNG UND INDUSTRIALISIERUNG” *
}

\author{
FORSCHUNGSANSÄTZE UND KONZEPTION
}

Das Thema „Schulbildung und Industrialisierung” gehört nicht zum klassischen Kanon der von den Historikern der Industrialisierung untersuchten Aspekte. Demgegenüber haben die empirischen Wirtschafts- und Sozialwissenschaften, im Zusammenhang mit der Faktoranalyse des wirtschaftlichen Wachstums und mit Blickrichtung auf die Zukunft entwickelter und unterentwickelter Volkswirtschaften, das Thema von verschiedenen Seiten in Angriff genommen. So spricht die Nationalökonomie vom ,human factor" oder Dritten Faktor neben Arbeit und Kapital und meint damit ,die natürlichen Fähigkeiten der Menschen eines Landes und die Erfolge und Anstrengungen der Bildungspolitik und den Ausbau der Forschung in vorausgegangenen Dezennien oder gar Jahrhunderten". ${ }^{1}$ Die neue Disziplin der Bildungsökonomie versucht u.a. Vorausschätzungen der Größe und Struktur des Bedarfs an qualifizierten Fachkräften und entwickelt „Strategies of Human Resource Development". ${ }^{2}$ Die Sozialpsychologie untersucht soziokulturelle Faktoren (Wertsysteme, psychische Dispositionen) und ihre Bedeutung für wirtschaftliches Wachstum, fragt direkt: „Does Education Accelerate Economic Growth?”3 Allgemein gilt, daß

* Der vorliegende Aufsatz ist Bestandteil der in der Historischen Kommissicn zu Berlin unter der Leitung von Priv. -Doz. Dr Otto Büsch laufenden Arbeiten zur Geschichte der frühen Industrialisierung im Wirtschaftsraum BerlinBrandenburg. Diese Arbeiten werden von derDeutschen Forschungsgemeinschaft als Schwerpunktprogramm gefördert. - Für methodische Anregungen bin ich Prof. Dr Rudolf Braun zu Dank verpflichtet; vgl. auch Rudolf Braun, Zur Einwirkung sozio-kultureller Umweltbedingungen auf das Unternehmerpotential und das Unternehmerverhalten, in: Wirtschafts- und sozialgeschichtliche Probleme der frühen Industrialisierung, hrsg. von Wolfram Fischer [Einzelveröffentlichungen der Historischen Kommission zu Berlin, Bd 1], Berlin 1968 , S. $247 \mathrm{ff}$.

1 Gottfried Bombach, Bildungspolitik und wirtschaftliche Entwicklung, in: Vortragsreihe des Deutschen Industrieinstituts, Jg. 14, Nr 12, 1964, S. 2.

${ }^{2}$ Frederick Harbison und Charles A. Myers, Education, Manpower and Economic Growth. Strategies of Human Resource Development, New York/Toronto/ London 1964.

${ }^{3}$ David C. McClelland, Does Education Accelerate Economic Growth? in: Economic Development and Cultural Change, 16, 1966, S. $257 \mathrm{ff}$. 
Schulbildung in Theorien des sozialen Wandels als wichtige Variable genannt wird, die den Prozeß der „sozialen Mobilisierung”, die Modernisierung der Gesellschaft, indiziert. ${ }^{1}$

Allen diesen Arbeiten und Forschungsansätzen ist gemeinsam, $\mathrm{da} \beta$ sie sich mit interkulturellen Vergleichen befassen und auf statistisches Material unseres Jahrhunderts stützen. Die starke Berücksichtigung unterentwickelter Länder in derartigen Untersuchungen gibt Veranlassung, die Frage nach dem Zusammenhang von Schulbildung und Industrialisierung auch in der Industrialisierungsgeschichte der westeuropäischen Länder im 18./19. Jahrhundert zu stellen und damit in den Kreis der Themen zur Geschichte der Industrialisierung aufzunehmen. Historische Fallstudien, die diese Fragen in den Mittelpunkt stellen, gibt es noch nicht. Einzelne Wirtschafts- und Sozialhistoriker sehen jedoch das Problem und liefern erste Beiträge zu seiner Lösung. So nennt z.B. Sidney Pollard unter den Mitteln, die dem frühen Unternehmer oder Management zur Verfügung standen, um die bekannten Schwierigkeiten der Fabriksdisziplin zu meistern, den Weg der Indoktrination eines neuen Arbeitsethos und bestimmter bürgerlicher Tugenden. ${ }^{2}$ David Landes versucht die Frage, warum die industrielle Führung gegen Ende des 19. Jahrhunderts von England auf Deutschland überging, mit der vergleichsweise rückständigen technologischen Entwicklung in England zu erklären, die ihrerseits u.a. eine Folge des Erziehungssystems gewesen sei. ${ }^{3}$ Für Deutschland haben Kurt Hinze ${ }^{4}$ und Ulrich Peter Ritter ${ }^{5}$ die Qualifizierung von Arbeitskräften in der Entstehungszeit der Industrie beschrieben.

1 Vgl. Karl W. Deutsch, Soziale Mobilisierung und politische Entwicklung, in: Theorien des sozialen Wandels, hrsg. von Wolfgang Zapf [Neue Wissenschaftliche Bibliothek, 31], Köln/Berlin 1969, S. 329ff., bes. S. 335; Daniel Lerner, Die Modernisierung des Lebensstils: eine Theorie, in: a.a.O., S. 362ff., bes. S. 365 ; zur politischen Wirkungsmöglichkeit von Bildungsinvestitionen vgl. Bert $F$. Hoselitz, Investment in Education and Its Political Impact, in: Education and Political Development, hrsg. von James S. Coleman, Princeton 1965, S. 541ff. Deutsche Ubersetzung: Bert F. Hoselitz, Wirtschaftliches Wachstum und Sozialer Wandel [Schriften zur Wirtschafts- und Sozialgeschichte, Bd 15], Berlin 1969, S. 227ff.

2 Sidney Pollard, Factory Discipline in the Industrial Revolution, in: Economic History Review, 16, 1963/64, S. 254ff.

${ }^{3}$ David S. Landes, Technological Change and Development in Western Europe. 1750-1914, in: The Cambridge Economic History of Europe, VI/1, Cambridge 1965 , S. $553 \mathrm{ff}$.

4 Kurt Hinze, Die Arbeiterfrage zu Beginn des modernen Kapitalismus in Brandenburg-Preußen 1685-1806. Bibliographisch vermehrte und verbesserte, mit einem Registeranhang versehene zweite Auflage. Mit einer Einführung von Otto Büsch [Veröffentlichungen der Historischen Kommission zu Berlin, Band 9, Neudrucke Band 1], Berlin 1963.

${ }^{5}$ Ulrich Peter Ritter, Die Rolle des Staates in den Frühstadien der Industriali- 
Die systematische Untersuchung des Themas "Schulbildung und Industrialisierung" erfordert eine umfassende Fragestellung. ${ }^{1}$ Geht man von der Institution der Schule aus, zeigen sich im wesentlichen drei Forschungsansätze: Eine soziologisch-historische Institutionengeschichte fragt nach Veränderungen im Schulsystem, neuen Schultypen, der Durchlässigkeit des Systems (Berechtigungswesen), Veränderungen im Benutzerkreis und nach der staatlichen Schulpolitik; eine statistisch-bildungsökonomische Untersuchung zeigt Wachstumskurven zur Schülerzahl (mit Rücksicht auf Bevölkerungswachstum und Schultyp), zur personellen und finanziellen Seite des Schulwesens; eine bildungsgeschichtlich-ideologiekritische Betrachtung untersucht das qualitative Angebot der Schulen (Lehrpläne, Schulbücher), fragt nach den vermittelten Kenntnissen, nach Wesen und Funktion der empfohlenen Verhaltensnormen, nach intendierter Weltanschauung. Die lückenlose Beantwortung dieser Fragen wird gleichwohl noch nicht ein Urteil über den Zusammenhang von Schulbildung und Industrialisierung zulassen. Man wird weiter fragen müssen nach der Stellung des Schulabsolventen im Wirtschaftsprozeß: Welchen Einfluß haben Bildung und Ausbildung auf den Arbeitsmarkt? Welche Selektionskriterien gelten für die Personalpolitik der Unternehmer? Sind Aufstiegschancen und Sozialprestige bildungsmäßig determiniert? Das methodische Grundproblem, die Bedeutung von Schulbildung als Faktor für die Industrialisierung abzuschätzen, wird freilich kaum schon hinreichend gelöst werden können; eine quantitative Erfassung des Beitrages des Erziehungswesens zum wirtschaftlichen Wachstum im 19. Jahrhundert, wie sie Knut Borchardt fordert, ${ }^{2}$ wird weiterhin Desiderat bleiben.

Der vorliegende Aufsatz greift aus der Fülle der Forschungsansätze einen heraus, der quellenmäßig und methodisch weitgehend Neuland betritt: die Schulbuchanalyse. Dieser Ausgangspunkt bedarf hinsichtlich seines Gegenstandes und des Erkenntnisziels einer gewissen Vorüberlegung. Schulbücher stellen eine verordnete $Z$ wangslektüre für Menschen einer Altersstufe dar, die einer dauerhaften Prägung be-

sierung. Die preußische Industrieförderung in der ersten Hälfte des 19. Jahrhunderts, Berlin 1961.

1 Vgl. Peter Lundgreen, Schulbildung und Frühindustrialisierung in Berlin/ Preußen. Eine Einführung in den historischen und systematischen Zusammenhang von Schule und Wirtschaft. Manuskript, erscheint 1970 in: Untersuchungen zur Geschichte der frühen Industrialisierung vornehmlich im Wirtschaftsraum Berlin-Brandenburg, hrsg. von Otto Büsch [Veröffentlichung der Historischen Kommission zu Berlin].

2 Knut Borchardt, Zum Problem der Erziehungs- und Ausbildungsinvestition im 19. Jahrhundert, in: Beiträge zur Wirtschafts- und Stadtgeschichte. Festschrift für Hektor Amman, Wiesbaden 1965, S. 390. 
sonders leicht zugänglich sind. Es scheint gerechtfertigt, diese Lektüre als ein Massenkommunikationsmedium zu begreifen, dem wesentliche Funktionen im Erziehungsprozeß zur normativen Daseinsbewältigung zukommen. Das gilt besonders für Lesebücher (z.T. noch für Geschichtslehrbücher), in denen die Kenntnisvermittlung hinter der Gesinnungsbildung, der Einübung in Verhaltens- und Denkmuster zurücktritt. Ohne auf die Frage eingehen zu müssen, wieweit diese Aussage auch heute noch gilt, ${ }^{1}$ kann ihre Gültigkeit für die dieser Studie zugrundeliegenden Lesebücher nachgewiesen werden.

Wenn nun seit Max Weber die Diskussion um den „kapitalistischen Geist" nicht verstummt ist, wenn anerkannt ist, daß die moderne industrielle Arbeitswelt bestimmte Arbeitstugenden in einer vorher nicht dagewesenen Art und Weise verlangt, dann liegt es nahe, die gesamtgesellschaftliche Durchsetzung des geforderten Verhaltensideals in dem Medium des Lesebuchs aufzuspüren. David C. McClelland ist diesen Weg gegangen;² er benutzt Lesebücher, um z.B. die Leistungsmotivation eines Volkes zu einer bestimmten Zeit zu messen; und Heinz Heckhausen spricht von McClellands „Geniestreich, Lesebuchgeschichten als Inhaltsmaterial heranzuziehen. Denn sie sind offensichtlich der eingedickte Niederschlag des von Pädagogen filtrierten nationalen Zeitgeistes..."3 Der Niederschlag dieses "nationalen Zeitgeistes” sei, so Heckhausen, „[in diesen Schuljahren] noch frei von Ideologie", und McClelland wählt aus eben diesem Grunde Lesebücher der untersten Schulklassen, ${ }^{4}$ um, wenn auch vielleicht nicht ideologiefreie, so doch gesamtgesellschaftlich verbindliche Wertordnungen, Verhaltensformen und Motivationen analysieren zu können.

Diese einfache Voraussetzung McClellands, daß die von ihm herangezogenen Lesebuchgeschichten für alle Kinder eines Landes Pflichtlektüre waren, läßt sich für Deutschland und das 19. Jahrhundert in dieser Form nicht machen. Hier muß vielmehr berücksichtigt werden, daB es keine allgemeine Grundschule gab, die alle sozialen Schichten erfaßt hätte. Die Volksschule brauchte lange, bis sie als Grundschule auch für Angehörige der „höheren Stände” genügend attraktiv

1 Vgl. Klaus Laermann, Das Lesebuch als gesellschaftliche Institution, in: alternative. Zeitschrift für Literatur und Diskussion, 45, Dezember 1965, S. $249 \mathrm{ff}$.

2 Vgl. David C. McClelland, The Achieving Society, New York 1961. Deutsche Úbersetzung: Die Leistungsgesellschaft. Psychologische Analyse der Voraussetzungen wirtschaftlicher Entwicklung, hrsg. von Ingeborg Y. Wendt und Gerd Fleischmann, Stuttgart 1966.

3 Siehe Heinz Heckhausen, Leistungsmotivation, in : Handbuch der Psychologie in 12 Bänden, 2. Band, Göttingen 1965, S. 621.

4 Siehe McClelland, Die Leistungsgesellschaft, S. 114. 
oder gar obligatorisch war und den Privatlehrer bzw. die schulgeldpflichtigen Vorbereitungsanstalten für die Gymnasien verdrängte. Die Realschulen, Ausdruck eines neuen Bildungsbedürfnisses, schlossen sich gleichfalls schon frühzeitig nach unten ab, um dann ihrerseits den Kampf um Gleichberechtigung mit dem humanistischen Gymnasium aufzunehmen. Auf diese Weise blieb das Volksschulwesen lange isoliert und klassenspezifisch. ${ }^{1}$ Eine Analyse historischer Schulbücher muß daher immer nach dem sozialen Herkommen derer fragen, die die Bücher benutzen, und im Auge behalten, da $\beta$ alle Feststellungen zunächst nur schichtenspezifisch gültig sind. Das führt zu einer ideologiekritischen Fragestellung, insofern schichtenspezifische Verhaltensmuster die bestehenden Herrschaftsverhältnisse erkennen lassen bzw. aus ihnen erklärt werden können.

Die vorliegende Studie stützt sich vorwiegend auf Volksschullesebücher, erfaßt damit eine potentielle Leserschicht, die zahlenmäßig sehr groß ist, aus der sich aber nur zum Teil die „Kader” der Industrialisierung rekrutieren. Welche Bedeutung jedoch auch die Volksschule im Qualifizierungsproze $ß$ für die moderne industrielle Arbeitswelt hatte, ${ }^{2}$ wird einleuchten, wenn man den disziplinarischen Aspekt des „,kapitalistischen Geistes” bedenkt, der den Unternehmern erst die notwendigen Arbeiter für eine rationelle Betriebsführung zur Verfügung stellte. ${ }^{3}$ Die Erkenntnisabsicht dieser Untersuchung läßt sich demnach mit der Frage formulieren, welche Verhaltensnormen das Volksschullesebuch in vor- und frühindustrieller Zeit indoktrinierte und welche Rückschlüsse sich daraus auf die intendierte Stellung des Volksschulabsolventen in einer Gesellschaft ergeben, die im Industrialisierungsprozeß steht.

\section{DIE ENTWICKLUNG DES LESEBUCHINHALTES}

Bei jeder vergleichenden Betrachtung von Schulbüchern, die bis ins 18. Jahrhundert zurückgeht, ist zunächst auf die Tatsache zu verweisen, $\mathrm{da} B$ in allen Schulen unterhalb der Gelehrtenschulen Bibel und Kate-

1 Ein ähnliches schichtenspezifisches Erziehungswesen im England des 18. Jahrhunderts beschreibt Sidney Pollard, Die Bildung und Ausbildung der industriellen Klassen Britanniens im 18. Jahrhundert, in: Jahrbuch für Wirtschaftsgeschichte, 1966 - Teil IV, S. $11 \mathrm{ff}$.

${ }^{2}$ Vgl. dazu Landes, Technological Change..., S. 567ff., und die Arbeiten von Pollard.

${ }^{3}$ Max Weber, Die protestantische Ethik. Eine Aufsatzsammlung, hrsg. von Johannes Winckelmann [Siebenstern Taschenbuch 53/54], München/Hamburg 1965, S. 184ff. 
chismus lange die einzigen Bücher für den Unterricht waren. Andachtsund Erbauungsbücher münzen diesen Stoff um, teils unter pädagogischen Gesichtspunkten (Fragebücher), teils um theologischen Richtungen der Zeit zu genügen. Friedrich Eberhard von Rochow, der mit seinem Kinderfreund 1776 zum erstenmal die „Lücke zwischen Fibel und Bibel"1 in einer der Auffassungsgabe des Kindes gemäBen Form schließt, ${ }^{2}$ schreibt in der Einleitung zu seinem Versuch eines Schulbuches $:^{3}$ „Außer dem Katechismus und der Heilsordnung fand ich kein Schulbuch für den Landmann und außer dem Inhalte dieser Bücher keine Wissenschaft, die man dessen Kinder lehrte."

So ist es verständlich, da $\beta$ die ersten „Lesebücher” nach dem Verständnis ihrer Autoren ein Kompendium der für die Schüler notwendigen und nützlichen „Wissenschaften" sind und hauptsächlich Realienkunde und Verhaltenslehre umfassen. Der ständischen Gliederung entspricht dabei die Unterscheidung der Lesebücher für Land- und Bürgerschulen. In beiden Schulen gibt es zunächst keine altersklassenspezifischen Lesebücher. Im 19. Jahrhundert beginnt diese Differenzierung mit einer Einteilung des Lesebuchs etwa in "leichtere" und „schwerere" Stücke. Von den Gelehrtenschulen dringt die Aufteilung nach Fächern in die „Vorbereitungsschulen zu den höheren Klassen der Gymnasien" ein und wird allmählich Ordnungsprinzip der Bürgerschulen. Damit werden die „Lesebücher” immer stärker zu Lehrbüchern nur noch des Sprachunterrichts. Freilich gilt für diesen auch jetzt noch, was eine Verfügung des brandenburgischen Schulkollegiums 1844 schreibt: ${ }^{4}$ „Den edelsten Lesestoff für die reifere Jugend bilden ausgewählte Abschnitte aus der heiligen Schrift und eine große Anzahl von Kirchenliedern."

Für die Zwecke der vorliegenden Untersuchung erfolgte die Auswahl unter den nicht eben zahlreich zur Verfügung stehenden Lesebüchern nach folgenden Gesichtspunkten: 1. Betrachtung von zwei Zeitpunkten (1780 und 1840), die als Querschnittjahre den Zustand der vor- und frühindustriellen Zeit erfassen sollen. 2. Differenzierung nach Land-

1 Friedrich Eberhard von Rochow, Der Kinderfreund. Ein Lesebuch zum Gebrauch in Landschulen (Erster Teil), Brandenburg/Leipzig 1776, in: Sämtliche pädagogische Schriften, hrsg, von Fritz Jonas und Friedrich Wienecke, Band I, Berlin 1907, S. 135.

${ }^{2}$ Vgl. Hermann und Heinz Weimer, Geschichte der Pädagogik [Sammlung Göschen, Band 145], Berlin 1958, S. 100.

3 Siehe Friedrich Eberhard von Rochow, Versuch eines Schulbuches für Kinder der Landleute, oder Unterricht für Lehrer in niedern und Landschulen, Berlin 1772, in: Sämtliche pädagogische Schriften, Band I, S. 4.

${ }^{4}$ Siehe Ludwig von Rönne, Das Unterrichtswesen des Preußischen Staates, I. Band: Das Volksschul-Wesen des PreuBischen Staates, Berlin 1855, S. 664. 
und Stadtschule. 3. Gesicherte Verbreitung und Benutzung der Lesebücher in einem identischen und wirtschaftsgeschichtlich relevanten Raum (Berlin/Brandenburg). ${ }^{1}$ Die Analyse stützt sich demnach im wesentlichen auf folgende Bücher: Friedrich Eberhard von Rochow, Der Kinderfreund. Ein Lesebuch zum Gebrauch in Landschulen (Erster Teil, Brandenburg/Leipzig 1776); Samuel Ludwig, Der Bürgerfreund, ein Lesebuch für Kinder in Bïrgerschulen (Berlin 1787); (Otto Schulz), Berlinisches Lesebuch für Schulen (Berlin 1840); Preußischer Kinderfreund. Ein Lesebuch für deutsche Volksschulen, zusammengestellt von A. E. Preuß und J. A. Vetter (Königsberg, 149. Aufl. ${ }^{2}$ 1865). Weitere Bücher wurden zur Ergänzung herangezogen, insbesondere K. E. Philipp Wackernagel, Deutsches Lesebuch (I. Teil, Stuttgart 1843).

Mit den Lesebüchern des ausgehenden 18. Jahrhunderts befinden wir uns in der Zeit der ersten Lesebücher überhaupt, wie schon das Rochow-Zitat andeutete. Peter-Martin Roeder nennt in seiner $\mathrm{Ge}$ schichte und Kritik des Lesebuchs der Höheren Schule (1961) zwei Motive, die es zur Entstehung von Lesebüchern kommen ließen: 1. „Sammlungen deutscher Dichtung” (Musterstücke für den oratorisch-literarischen Unterricht); 2. „Prosabeiträge aus den verschiedensten Bereichen des Wissens, sogenannte ,Realstoffe'."3 Wenn man hinzunimmt, daß die literarische Bildung Vorrecht des gehobenen Bürgerstandes war und Dichtung in den Volksschullesebüchern bis in die zweite Hälfte des 19. Jahrhunderts eine völlig untergeordnete Rolle spielte, ${ }^{4}$ so ist damit der Lesebuchinhalt der hier betrachteten Volksschullesebücher bereits grundsätzlich charakterisiert. Ein „enzyklopädischer" Zug kennzeichnet vor allem das frühe Bürgerschullesebuch, wie das Inhaltsverzeichnis von Ludwigs Bürgerfreund (1787) erkennen läßt:

1 Vgl. Dietrich Rittershausen, Beiträge zur Geschichte des Berliner ElementarSchulwesens. Von der Reformation bis 1836, in: Märkische Forschungen, IX, 1865, S. 174 ; Rönne, Volksschul-Wesen..., S. 664f.; Josef Antz, Das Lesebuch, in: Handbuch der Erziehungswissenschaft, IV. Teil, Band 2: Die deutschsprachliche Jugendbildung in ihren Grundlagen, München 1932, S. 199 ff.; Ferdinand Bünger, Entwicklungsgeschichte des Volksschullesebuchs, Leipzig 1898, S. 127ff., S. $341 \mathrm{ff}$.

${ }^{2}$ Die 1. Auflage des Preußischen Kinderfreundes von 1836 war mir leider nicht zugänglich. Nach Bünger, Entwicklungsgeschichte des Volksschullesebuchs, S. 351ff., erfuhr der Preußische Kinderfreund trotz seiner langen Lebensdauer und hohen Auflage nur leichte Verbesserungen von der Hand des seinen Freund PreuB fast ein halbes Jahrhundert überlebenden Mitverfassers Vetter.

${ }^{3}$ Siehe Peter-Martin Roeder, Zur Geschichte und Kritik des Lesebuchs der höheren Schule, Weinheim/Bergstr. 1961, S. 11.

4 Siehe a.a.O., S. $43 f$. 
1. Von dem Menschen

2. Von Sonne, Mond, Erde, Wasser, Luft usw.

3. Von den Tieren

4. Von den Pflanzen

5. Von den Mineralien

6. Von Nahrungsmitteln, Kleidung, Wohnung, Hausgeräten und Arzneien
7. Nützliche Erzählungen

8. Biblische Erzählungen

9. Gute Lehren

10. Gute Sprichwörter

11. Einige Aufsätze, die im gemeinen Leben oft vorkommen

12. Briefe

Dabei fühlen sich die ersten Lesebuch-Autoren noch genötigt, die Einführung der Realienkunde in den bis dahin weitgehend von Bibel und Katechismus beherrschten Unterricht zu rechtfertigen. Rochow, der die Realien in seinem Versuch eines Schulbuches, einer Didaktik für die Hand des Lehrers, behandelt, nimmt den physiokratischen Standpunkt ein, wenn er schreibt: ${ }^{1}$ „Ein Landesherr, der die wichtige Wahrheit glaubt, daB im Ackerbau die Grundkraft des Staates liegt, wird mit den besten Edikten zur Verbesserung tauben Ohren predigen, wenn er nicht für die bessere Einrichtung der Schulen zur Bildung der Gemüter in der Jugend durch Unterricht in den nützlichsten ökonomischen Erkenntnissen Sorge trägt." Junker, dessen Handbuch der gemeinnützigsten Kenntnisse für Volksschulen in den Berliner Schulen als Lehrbuch der Naturgeschichte diente, ${ }^{2}$ tadelt die Monotonie des Religionsunterrichts, seine mangelnde Verbindung mit den „Werken Gottes” und dem wirklichen Leben, um zu fordern: ${ }^{3}$,Wenn man... mit dem Unterricht von Gott die Betrachtung der Natur, und mit dem Unterrichte von dem Willen Gottes die Betrachtung des wirklichen Lebens mehr verbände, so müßte er notwendig teils angenehmer, teils praktischer werden."

„Betrachtung der Natur” - „Betrachtung des wirklichen Lebens”, diese Unterscheidung kann als Einteilungsprinzip für die Lesebuchinhalte dienen: Realienkunde (im engeren Sinne, als „Weltkunde”) und Verhaltenslehre. Roeder rechnet die typischen Exempel zur Verhaltenslehre (,Tugenden”), wie sie diese Lesebücher enthalten, zu

1 Siehe Rochow, Versuch eines Schulbuchs..., in : Sämtliche pädagogische Schriften, Band I, S. 7.

${ }^{2}$ Vgl. Rittershausen, Beiträge zur Geschichte des Berliner Elementar-Schulwesens,, S. 274.

${ }^{3}$ F. A. Junker, Handbuch der gemeinnützigsten Kenntnisse für Volksschulen. Beim Unterricht als Materialien und bei Schreibübungen als Vorschriften zu gebrauchen, I. Teil, 6. Aufl., Halle 1803, S. 13. 
den Realstoffen, ${ }^{1}$ und er dürfte damit den Auffassungen der Aufklärung über Lernbarkeit und Nützlichkeit der Tugenden Rechnung tragen. Für unsere Zwecke erscheint es jedoch sinnvoll, eine Trennung vorzunehmen, denn die folgende Untersuchung stützt sich weitgehend auf diejenigen Teile der Lesebücher, die Verhaltenslehre zum Inhalt haben, und dabei ist interessant, wie stark dieser Aspekt repräsentiert ist.

Rochows Kinderfreund (1776), der im Gegensatz zu Ludwigs Bürgerfreund (1787) keine Gliederung nach Sachgebieten aufweist, bietet Realienkunde in Gesprächen oder eingekleidet in Geschichten an, räumt ihr aber nur etwa halb soviel Raum ein wie Ludwig, der systematisch natur- und kulturkundliche Bereiche vorstellt (Kap. 1-6, 11-12). Das gleiche Verhältnis in modifizierter Form läßt sich für die Lesebücher von 1840 feststellen. Preuß/Vetter bringen naturkundliche Beschreibungen und „Begriffserklärungen” inmitten ihrer Geschichten. Schulz hat es zwar nicht mehr nötig, so stark wie Ludwig der „enzyklopädischen" Tendenz des städtischen Lesebuchs zu folgen, weil sich inzwischen ein realkundlicher Fachunterricht zu etablieren beginnt, bietet aber gleichwohl mehr Realienkunde (Kap. 6, 8) als Preuß/ Vetter. Sein Inhaltsverzeichnis zeigt zugleich mit Kap. 7 die Intention des literarischen Lesebuchs, wenn auch noch die Verhaltenslehre (Kap. 2-6) im Vordergrund steht:

1. Gebete und Gedichte geistlichen Inhalts

2. Denksprüche

\section{Das güldne $\mathrm{ABC}$}

4. Poetische Fabeln und Erzählungen
5. Fabeln und Erzählungen in Prosa

6. Aufsätze, Erzählungen und Beschreibungen verschiedenen Inhalts

7. Natur, Gemüt und Vaterland in Gedichten

8. Zur Naturkunde

Wenn man allerdings berücksichtigt, daß Preuß/Vetter ihrem Kinderfreund noch 1865 ,auf den Wunsch einiger Herren Geistlichen und Schulmänner... mit Rücksicht auf unsere Landschulen"2 einen Anhang beifügen, der Stoffe zur Erdkunde, Geschichte, Naturlehre und -geschichte, zur Anatomie, über Gifte und Kräuter, zur Ersten Hilfe sowie Rechenaufgaben enthält, wird man vielleicht von einer Phasenverschiebung zwischen Stadtschule (Ludwig, 1787) und Landschule (Preuß/Vetter, 1865) sprechen können, was die Ausrichtung

1 Siehe Roeder, Geschichte und Kritik des Lesebuchs..., S. 11.

2 Siehe Preuß/Vetter, Preußischer Kinderfreund..., S. 239. 
des Lesebuchinhalts am realkundlichen Interesse betrifft. Der Anteil, den die Lesebücher (bzw. der Schulunterricht) der Realienkunde einräumen, dürfte jedenfalls das wachsende Bedürfnis einer Gesellschaft nach Qualifizierung durch Kenntnisvermittlung erkennen lassen und auf der Ebene der Volksschule das Korrelat zu den Bemühungen der neuen Fachschulen darstellen, ohne freilich zunächst und auf lange Sicht dem damit verbundenen Anspruch zu genügen. Es kann hier nicht näher auf die Geschichte des Realienunterrichts eingegangen, sondern nur gezeigt werden, in welchem Kontext diejenigen Teile des Lesebuchinhalts stehen, denen die vorliegende Studie ihr Hauptaugenmerk zuwendet, wobei bereits der Kontext auf die Berechtigung der theoretischen Position verweist, die das Lesebuch als gesellschaftliche Institution und Kommunikationsmedium für den Sozialisationsprozeß begreift.

\section{QUANTITATIVE INHALTSANALYSE DER VERHALTENSLEHRE}

Neben der Realienkunde (Sachkunde) steht die Verhaltenslehre (Wertkunde); sie zeigt sich in allen Lesebüchern in verschiedenen literarischen Formen, besonders als „Nützliche Erzählungen” (Geschichten) und „Nützliche Lehren”, als „Denksprüche”, ,,Sprichwörter”, „Regeln”. Aus Gründen der Vergleichbarkeit mit literarisch anspruchsvolleren Texten der späteren Zeit, aber auch, um den verschiedenen Methoden der Inhaltsanalyse die gleichen Einheiten zugrundezulegen, beschränkt sich die Untersuchung der Verhaltenslehre auf den literarischen Typus „Erzählung”, „Geschichte”. Man könnte nun meinen, es hier mit Literatur zu tun zu haben, und versucht sein, literaturwissenschaftliche Methoden zur Schulbuchanalyse heranzuziehen. Solche Versuche werden bisweilen gemacht, ${ }^{1}$ wobei fraglich bleibt, ob man das Lesebuch damit in seinem Selbstverständnis erfaßt und seine Funktion berücksichtigt. Nach Wolfgang Preibusch ist das Lesebuch mit den Methoden der literatur-kritischen Wissenschaften überhaupt nicht untersuchbar und faßbar, ${ }^{2}$ weil die Herausgeber von Lesebüchern ihre Aufgabe „nicht in der Schaffung einer Literaturstichprobe, sondern in der mühevollen Suche nach kleinen Informationseinheiten" sehen, „die bestimmte Interaktionsfolgen zeigen, mit bestimmten Frustrationen und Gratifikationen."3 Kultur-

1 Vgl. z.B. Walther Killy, Zugelassen zum Gebrauch in Schulen, in: Neue Deutsche Hefte, 3, 1956/57, S. 475ff.

${ }^{2}$ Siehe Wolfgang Preibusch, Experimentelle Untersuchung über die Beziehung der Inhalte von Lesebüchern zur Erinnerungs- und Interessenlage von Schülern (Abschlußarbeit der Pädagogischen Hochschule Berlin) 1962 (Ms.), S. 38 (Rezension in: alternative, 45, Dezember 1965, S. 258 f.).

${ }^{3}$ Siehe a.a.O., S. 81 . 
anthropologische und sozialpsychologische Kategorien müssen daher herangezogen werden, um das Lesebuch adäquat theoretisch einzustufen. ${ }^{1}$ Das führt zu folgender These: „Das Lesebuch ist eine sekundäre" (weil schichten-, gruppen- und rollenspezifische) „Institution; sie dient der Kommentierung und Interpretation von Institutionen. Sie fördert bestimmte sprachliche Wendungen und Begriffe, die ... zu spezifischen Denkmustern führen. Da das Denken und Erleben von Konflikten, aber auch Problemlösung und Befriedigung eine Frage der Verbalisierung ist, läuft die geistige Bewältigung der sozialen Realität weitestgehend mit Quantität und Qualität der erlernten Denkmuster zusammen."2

Die erneute Beleuchtung der hier zugrundegelegten theoretischen Position macht das Lesebuch als Quelle für den Historiker nicht weniger interessant, sondern steigert womöglich noch den Quellenwert, fordert allerdings auch heraus zur Reflexion der Methoden, die dieser Quelle adäquat sind. Es liegt nun nahe, nach einer kulturanthropologisch-sozialpsychologischen Einstufung des Lesebuchs mit den Mitteln eben dieser Wissenschaften die Analyse zu unternehmen, zumindest diesen Weg auch einmal zu gehen, und zwar unter besonderer Berücksichtigung der hier interessierenden Fragen nach der Beziehung des Lesestoffs zur Wirtschaftswelt. Wir wollen das anhand der von McClelland entwickelten Verfahren versuchen, denn auch hier werden Lesebuchgeschichten in der oben skizzierten theoretischen Bedeutung aufgefaßt: „Sie beschreiben eingebildete Situationen, manchmal phantastische (aus der Welt der Feen, Riesen und Zwerge) und manchmal realistische (aus dem täglichen Leben), aber die Absicht ist immer dieselbe, nämlich den Kindern einen interessanten und instruktiven Lesestoff zu geben. In diesem Sinne sind die Lesebuchgeschichten ,projektiv', sie reflektieren die Motive und Werte der Kultur in der Art, wie sie erzählt werden oder in ihren Themen oder Handlungsabläufen."3

$\mathrm{McClelland}$ wertet dementsprechend Lesebuchgeschichten als Indikatoren für Veränderungen aus, die sich in Motivation und Sozialstruktur einer Gesellschaft zeigen. Er geht dabei von der Motivationstheorie aus, und zwar zunächst individualpsychologisch. Die hier gewonnene Möglichkeit, das „Bedürfnis nach Leistung” (n Achievement) ${ }^{4}$ als ein elementares Grundmotiv des Menschen experimentell zu

1 Siehe a.a.O., S. 39.

2 Siehe a.a.O., S. 53.

3 Siehe McClelland, Die Leistungsgesellschaft, S. 114.

"Der Ausdruck „n Achievement" ist ein terminus technicus, der anzeigt, daß es sich hier um eine mit einer bestimmten Methode quantitativ bestimmbare 
isolieren und quantitativ zu bestimmen, ${ }^{1}$ veranlaßt $\mathrm{zu}$ der sozialpsychologischen Frage, ,welche Konsequenzen es haben mag, wenn in einer Gesellschaft eine größere Zahl von Menschen mit einem hohen ,Bedürfnis nach Leistung' auftreten."2 Gewisse Parallelen zwischen der Orientierung von Testpersonen mit hoher Leistungsmotivation und der Haltung der protestantischen Unternehmer zu Berufserfüllung und Profit, wie sie Max Weber beschrieben hat, ${ }^{3}$ führen zu der Hypothese: Hohes durchschnittliches "Leistungsbedürfnis" führt zu größerem wirtschaftlichen Fortschritt. Zur Prüfung dieser Hypothese bedarf es zunächst eines Mediums, das den im Individualtest (TAT) verwendeten Phantasiegeschichten vergleichbar ist und als Grundlage zur Messung der Leistungsmotivation einer Gesellschaft dienen kann. McClelland wählt aus den oben angeführten Gründen Lesebuchgeschichten für diesen Zweck aus. In einem interkulturellen Vergleich ergibt sich, daß für 1925 und 1950 jeweils hohes Niveau an „Leistungsbedürfnis”“ und größere wirtschaftliche Entwicklung ${ }^{5}$ positiv korrelieren. ${ }^{6}$

Das Verfahren zur Messung der Leistungsmotivation einer Gesellschaft anhand von Lesebuchgeschichten bedarf einer kurzen Erläuterung. Die Geschichten werden unter dem Gesichtspunkt analysiert, wieweit sie leistungsbezogene Elemente enthalten. Als Leitfaden dient folgendes Schema (in vereinfachter Form): Die handelnde

Größe (need for achievement) handelt, im Unterschied zu der qualitativen, aber unbestimmten Rede vom Leistungsbedürfnis eines Menschen. Die deutsche Ubersetzung bietet daher, in Anlehnung an Heinz Heckhausen, analog den Terminus „b Leistung” (Bedürfnis nach Leistung) an. In dem vorliegenden Aufsatz soll der gemeinte technische Charakter der gemessenen GröBe „Leistungsbedürfnis" durch Anführungsstriche angedeutet werden.

1 Vgl. David C. McClelland, John W. Atkinson, Russel A. Clark und Edgar L. Lowell, The Achievement Motive, New York 1953.

2 Siehe McClelland, Die Leistungsgesellschaft. Vorbemerkungen der Herausgeber (Gerd Fleischmann), S. 21.

${ }^{3}$ Siehe McClelland, Die Leistungsgesellschaft, S. 89ff.

4 Gemessen an je 21 Lesebuchgeschichten einer Nation für die beiden Zeiträume.

5 Gemessen an der positiven oder negativen Abweichung der Zuwachsrate der Elektrizitätserzeugung von dem auf Grund einer Regressionsgleichung erwarteten Wert.

6 Vgl. McClelland, Die Leistungsgesellschaft, S. 133ff.; die Anwendung dieser Untersuchungsmethode auf monokulturelle Fälle haben einige Forscher vorgenommen: Juan B. Cortés, The Achievement Motive in the Spanish Economy between the 13th and 18th centuries, in: Economic Development and Cultural Change, 9, 1960/61, S. 144ff.; Norman M. Bradburn und David E. Berlew, Need for Achievement and English Industrial Growth, in: Economic Development and Cultural Change. 10, 1961, S. 8ff.; Richard de Charms und Gerald H. Moeller, Values expressed in American Children's Readers, in: Journal of abnormal and social Psychology, 64, 1962, S. 136ff. 
Person hat das Verlangen, ein Ziel zu erreichen; sie hat bestimmte Vorstellungen über Erfolg oder Mißerfolg, begleitet von entsprechenden Affekten; sie stößt auf bestimmte Widerstände, überwindet diese mit eigener oder fremder Hilfe. Jedes dieser leistungsbezogenen Elemente einer Geschichte wird einmal gezählt, sofern es überhaupt auftritt; die Summe der Elemente stellt das Niveau an „Leistungsbedürfnis” einer Geschichte dar; das arithmetische Mittel aller Niveau-Werte der einzelnen Geschichten bildet das Niveau an „Leistungsbedürfnis” der durch die Geschichten repräsentierten Gesellschaft. ${ }^{1}$ Wendet man diese Methode zur Messung des Niveaus an „Leistungsbedürfnis” auf je 24 Lesebuchgeschichten ${ }^{2}$ von Rochow, Ludwig, Schulz und Preuß/ Vetter an, so kann man eine Verdoppelung des Niveaus an „Leistungsbedürfnis" zwischen 1780 und 1840 konstatieren (Tabelle 1).

\section{Tabelle 1}

Niveau an ,Leistungsbedürfnis” 1780 und 1840

1780

\begin{tabular}{|c|c|c|c|}
\hline Lesebuch & $\begin{array}{l}\text { Niveau an "Lei- } \\
\text { stungsbedürfnis" }\end{array}$ & Lesebuch & $\begin{array}{l}\text { Niveau an "Lei- } \\
\text { stungsbedürfnis" }\end{array}$ \\
\hline Rochow (1776) & 0,96 & Schulz (1840) & 1,62 \\
\hline Ludwig (1787) & 0,83 & $\begin{array}{l}\text { Preuß/Vetter } \\
(1865)\end{array}$ & 1,83 \\
\hline
\end{tabular}

Durchschnitt

0,89

Durchschnitt

Dieses Ergebnis läßt eine Einordnung in McClellands Befunde zu, $\mathrm{da} B$ ein Ansteigen des Niveaus an „Leistungsbedürfnis" einem wirtschaftlichen Wachstum vorausgeht, wobei die Frage einer wie immer meßbaren Wachstumskurve der deutschen oder Berliner Wirtschaft hier offen bleiben muB. Die Interpretation dieser Korrelation bedarf dagegen noch der Überlegung, wobei mögliche psychologische ${ }^{3}$ und wirtschaftstheoretische ${ }^{4}$ Einwände zu McClellands Methode noch weitere, hier nicht mehr aufgegriffene Diskussionen nötig machen.

1 Vgl. McClelland (et al.), The Achievement Motive, S. $109 \mathrm{ff}$.

${ }^{2}$ Die Geschichten sind nicht ausgewählt. Das Problem der Repräsentanz der 24 Geschichten in statistischer Hinsicht stellt sich nicht, weil die Zahl der in der Stichprobe berücksichtigten Geschichten so hoch ist, daß sie im ungünstigsten Fall immer noch nahezu 50\% der Gesamtmenge ausmacht.

${ }^{3} \mathrm{Vgl}$. McClelland, Die Leistungsgesellschaft, Vorbemerkungen der Herausgeber (Ingeborg Y. Wendt), S. 16ff.

Vgl. a.a.O., Vorbemerkungen der Herausgeber (Gerd Fleischmann), S. $28 \mathrm{ff}$. 
McClelland interpretiert die Korrelation von „Leistungsbedürfnis” und Wirtschaftswachstum als Kausalzusammenhang und sieht als sozialen Mechanismus zur Umsetzung des „Leistungsbedürfnisses” in wirtschaftiche Leistung, als Zwischenglied, den Unternehmer. ${ }^{1}$ Die Ursachen für ein Steigen und Fallen des "Leistungsbedürfnisses" findet er in den Kindererziehungspraktiken und der Art der religiösen Einstellung. ${ }^{2}$ Unter diesen Voraussetzungen erscheint es konsequent, die Lesebücher nicht nur als Indikator für "Leistungsbedürfnis” zu benutzen, sondern auch als ein Indoktrinationsmedium für dieses anzusehen. Ohne jedoch etwas über den Wirksamkeitsgrad solcher Medien der Kommunikation zu wissen, warnt uns auch noch ein anderer Umstand vor einer Uberbewertung der konstatierten Steigerung des Niveaus an „Leistungsbedürfnis”. Denn selbst wenn die Unternehmer als "Zwischenglieder" sich in nennenswertem Umfang aus den sozialen Schichten rekrutiert haben sollten, für die die hier zugrundegelegten Volksschullesebücher spezifisch sind, so steht, wie noch zu zeigen sein wird, die in diesen Lesebüchern propagierte Verhaltenslehre in einigen Gegensatz zu der „unternehmerischen Rolle"3 oder dem Weberschen Unternehmerverhalten. Damit wird die kausale Interpretation der Beziehung zwischen „Leistungsbedürfnis” und Wirtschaftswachstum zumindest fraglich in dem Sinne, daß man genauer untersuchen muß, welche Funktion bestimmte Veränderungen für die verschiedenen sozialen Schichten haben, eine Frage, die mit quantitativen Methoden nicht $\mathrm{zu}$ beantworten ist.

Ist hohes "Leistungsbedürfnis" somit höchstens als notwendige, wenn auch kaum als hinreichende Bedingung wirtschaftlichen Wachstums anzusprechen, so versucht McClelland, eine weitere hypothetische Voraussetzung, nämlich die spezifische Beschaffenheit einer für wirtschaftliches Wachstum günstigen Sozialstruktur, inhaltsanalytisch zu verifizieren. Aus der Analyse der Handlungsabläufe ${ }^{4}$ der Lesebuchgeschichten gewinnt er Daten (Merkmalshäufigkeiten), die er mit den Idealnormen für traditionelle und „moderne” Sozialstruktur, wie sie Hoselitz im AnschluB an Parsons entworfen hat, ${ }^{5}$ in Beziehung setzt. ${ }^{6}$ Die Hypothese lautet: Länder mit schnellerem wirtschaftlichen

1 Vgl. a.a.O., S. 201 if.

2 Vgl. a.a.O., S. $285 f$ f.

3 Vgl. a.a.O., S. 204.

4 Die Analyse des Handlungsablaufs fragt nach Status, Beeinflussung, Reaktion, Erfolg, Mitteln und Motiven usw. der führenden Person einer Geschichte („ego") sowie des Gegenüber, des Gegenspielers oder der Welt (,alter").

${ }^{5}$ Vgl. Bert F. Hoselitz, Social Structure and Economic Growth, in: Sociological Aspects of Economic Growth, Glencoe/IIl. 1960, S. 23ff. Deutsche Ubersetzung: Bert F. Hoselitz, Wirtschaftliches Wachstum und Sozialer Wandel, S. 11ff.

- Vgl. McClelland, The Achieving Society, S. 171ff. 


\section{Tabelle 2}

Hypothetische Beziehungen zwischen Sozialstruktur (Normenalternativen) und Lesebuchinhalt (Merkmalshäufigkeiten)

Normen einer „modernen" Gesellschaft [Sozialstruktur] (gegenüber einer traditionalistischen)
Merkmalshäufigkeiten in Geschichten aus Ländern mit schnellerem wirtschaftlichen Wachstum (gegenüber solchen mit langsamerem)
1. Ablehnung der Verbindlichkeit traditioneller Institutionen

a. universalist. Verhaltensnormen [gleiches Gesetz für alle] (gegenüber partikularist. Verhaltensnormen [Privilegien für einzelne]) b. funktionsspezifische Beziehungen untereinander [z.B. Arzt-Patient] (gegenüber diffusen, polyfunktionalen Beziehungen [z. B. Meister-Geselle im alten Handwerk]) c. erworbener Status [auf Grund von Leistung] (gegenüber zugeschriebenem Status [z.B. auf Grund der Herkunft])

d. kollektive Orientierung der Person (gegenüber selbstorientiertem [elitärem] Denken)

2. Person ist neutral gegenüber Affekten und Impulsen, beherrscht

3. Rationalität und Planung (gegenüber Magie)

4. Optimismus und Vertrauen in die eigene Fähigkeit, die Natur zu besiegen

5. Betonung materieller Bedürfnisse gegenüber anderen Anliegen
1. Institutioneller Druck auf „ego" seltener

2. Ebenbürtiger Status von „ego" häufiger

3. „ego" hat vertragsähnliche Beziehungen, motivierte Beweggründe häufiger

4. „ego" hat auf Leistung beruhenden Status häufiger

5. Ebenbürtiger Druck auf "ego" häufiger

6. Selbstinteresse, Selbstachtung und Mildtätigkeit als Motive für ,ego" seltener

7. Impulskontrolle häufiger

8. Harte Arbeit, Intelligenz als Instrumente (gegenüber Täuschung, Magie) häufiger

9. Schicksal und Magie als Einflüsse auf "ego" seltener

10. Mensch siegt über Natur häufiger

11. ,ego" ist erfolgreich häufiger

12. Biologische Bedürfnisse als Motive für "ego" häufiger

13. Materielle Anreizeals Druckmittel auf „ego" häufiger

14. Unpersönlicher Druck (Natur) veranlaßt Zusammenarbeit häufiger 
Wachstum haben eine „moderne” Sozialstruktur, die sich in den Lesebuchgeschichten niederschlägt. Die Art dieses hypothetischen Niederschlags veranschaulicht Tabelle $2 .^{1}$

McClelland findet, wiederum in einem interkulturellen Vergleich für 1925 und 1950, daß schnelles wirtschaftliches Wachstum jeweils mit einer statistisch signifikanten größeren Häufigkeit des Auftretens der Merkmale 1, 3, 5, 8 und 14 in den Lesebuchgeschichten der entsprechenden Länder korreliert, und sieht damit die entsprechenden Hypothesen bestätigt. Auch hier führt die Anwendung dieser Methode der Inhaltsanalyse auf die Lesebücher von 1780 und $1840 \mathrm{zu}$ einem vergleichbaren Ergebnis, wobei der monokulturelle Längsschnitt ${ }^{2}$ in unserem Fall den Wandel der Sozialstruktur parallel zur wirtschaftlichen Entwicklung zu erkennen gäbe. Die Auszählung der Merkmale 1-14 in je 24 Geschichten der mehrfach benutzten Lesebücher ist in Tabelle 3 zusammengefaßt.

\section{Tabelle 3}

Sozialstruktur im Spiegel der Lesebücher 1780 und 1840

\begin{tabular}{|c|c|c|c|c|c|c|c|c|c|c|c|c|c|c|}
\hline Merkmale: & 1 & 2 & 3 & 4 & 5 & 6 & 7 & 8 & 9 & 10 & 11 & 12 & 13 & 14 \\
\hline $\begin{array}{l}\text { Rochow } \\
\text { (1776) }\end{array}$ & 9 & 3 & 7 & 3 & 2 & 16 & 0 & 4 & 2 & 0 & 7 & 1 & 0 & 1 \\
\hline $\begin{array}{l}\text { Ludwig } \\
\text { (1787) }\end{array}$ & 9 & 6 & 6 & 3 & 3 & 11 & 0 & 4 & 2 & 1 & 6 & 0 & 0 & 2 \\
\hline $\begin{array}{l}\text { Häufigkeit } \\
\text { für } 1780\end{array}$ & 18 & 9 & 13 & 6 & 5 & 27 & 0 & 8 & 4 & 1 & 13 & 1 & 0 & 3 \\
\hline $\begin{array}{l}\text { Schulz } \\
(\mathbf{1 8 4 0 )}\end{array}$ & 3 & 9 & 17 & 2 & 4 & 12 & 0 & 9 & 1 & 0 & 10 & 2 & 3 & 2 \\
\hline $\begin{array}{l}\text { Preuß/Vetter } \\
\text { (1865) }\end{array}$ & 2 & 8 & 14 & 1 & 5 & 16 & 4 & 8 & 0 & 3 & 13 & 3 & 0 & 1 \\
\hline $\begin{array}{l}\text { Häufigkeit } \\
\text { für } 1840\end{array}$ & 5 & 17 & 31 & 3 & 9 & 28 & 4 & 17 & 1 & 3 & 23 & 5 & 3 & 3 \\
\hline
\end{tabular}

Unterwirft man die Häufigkeitsverteilung der Merkmale für 1780 und 1840 einem Signifikanztest, so gelten mit einer Irrtumswahrscheinlichkeit von 1\% die Hypothesen 1 und 3, mit einer solchen von

1 Siehe a.a.O., Table 5.4, S. 173. Die deutsche Ausgabe hat dieses Kapitel nur in einer kurzen Inhaltsangabe aufgenommen, aus der ich einige deutsche Ausdrïcke übernehme.

2 $\mathrm{Zu}$ monokulturellen Untersuchungen vgl. die S. 96, Anm. 6 zitierte Literatur. 
$6 \%$ die Hypothesen 8 und 11 als gesichert in dem Sinne, daß die Häufigkeit der Merkmale 1, 3, 8 und 111840 gegenüber 1780 einen statistisch signifikanten Unterschied aufweist. ${ }^{1}$ Die eigentlichen Probleme liegen wiederum in der Deutung des Befundes. Da sich unser Ergebnis weitgehend mit McClellands Ergebnissen deckt, folgen wir zunächst seiner Interpretation:2

1. „Harte Arbeit” (Hyp. 8) muß notwendigerweise ein typisches Verhalten für eine Gesellschaft sein, die ein relativ hohes Niveau an „Leistungsbedürfnis” hat. Insofern entsprechen sich unsere Untersuchungsergebnisse: Verdoppelung des "Leistungsbedürfnisses”, Verdoppelung der Häufigkeit von ,harter Arbeit” zwischen 1780 und 1840. McClelland rechnet daher Hyp. 8 zum "Achievement Syndrome", das in seiner Gesamtheit (- wir dürfen auch Hyp. 11 hier mitzählen -) eine "moderne" Sozialstruktur charakterisiert, die ihrerseits eine wichtige, womöglich notwendige Voraussetzung für wirtschaftliches Wachstum ist.

2. Der institutionelle Druck, die Bedeutung von Traditionen gehen zurück (Hyp. 1); spezifische zwischenmenschliche Beziehungen nehmen größeren Raum ein (Hyp. 3); Bedürfnisse und deren Befriedigung stellen menschliche Beziehungen unter Gleichgestellten her (Hyp. 5): Die Gesellschaft ist offener; als Regulator der Beziehungen gelten nicht mehr vorgeschriebene Traditionen, sondern Nachfrage, Ưberzeugungen und Wünsche des ,anderen”. - Auf die Frage, warum es sinnvoll erscheint, eine Beziehung zwischen dieser Art von Gesellschaft bzw. ihrer Sozialstruktur und wirtschaftlicher Entwicklung zu sehen, gibt McClelland drei Antworten. Einmal wird die Unsicherheit beim Verlassen traditioneller Normen im Prozeß der Meinungsbildung beseitigt durch eine neue Autorität, die öffentliche Meinung, als Ausdruck des ,anderen”. Sie erleichtert die Etablierung funktionaler Verhältnisse. Zum zweiten ist die öffentliche Meinung flexibler, kann auf wechselnde Bedingungen schneller reagieren als Traditionen, kann schneller mobilisiert werden, um nötige Neuerungen zu unterstützen. Für den einzelnen führt Fachwissen zur Spezifikation der Rolle und Unabhängigkeit von Status und Loyalität. Und drittens können Marktunvollkommenheiten, die auf partikularistischen Normen beruhen (z.B. Beschränkung der Teilnehmerzahl, unterschiedliche

1 Vgl. das $\mathrm{X}^{2}$-Näherungsverfahren bei Graf/Henning/Stange, Formeln und Tabellen der mathematischen Statistik, 2. Aufl., Berlin/Heidelberg/New York 1966, S. 89ff. - Für statistische Beratung bin ich Dr Peter Czada zu Dank verpflichtet.

2 Vgl. McClelland, The Achieving Society, S. 191ff.; zu den nicht bestätigten Hypothesen vgl. a.a.O., S. 178ff. 
Behandlung bestimmter Gruppen wie Untergebene, Freunde usw.), beseitigt werden, indem die öffentliche Meinung eine „Marktmoral” fördert und Verstöße gegen sie (schlechte Qualität, ungerechtfertigte Preise, Bevorzugung und Benachteiligung) anprangert.

McClelland sieht damit neben dem "Bedürfnis nach Leistung” einen weiteren meßbaren Faktor, der mit wirtschaftlicher Entwicklung in Zusammenhang steht, nämlich die „Einstellung und innere Ausrichtung auf die Meinung anderer", 1 die .Außenlenkung” (OtherDirectedness). Die Anwendung der von ihm entwickelten Methoden auf Lesebücher des späten 18. und frühen 19. Jahrhunderts führt zu überraschenden Parallelen: Im interkulturellen Querschnitt des 20. Jahrhunderts wie im monokulturellen Längsschnitt des 19. Jahrhunderts korrelieren hohes „Leistungsbedürfnis” und stärkere „Außenlenkung" mit wirtschaftlichem Wachstum. Insofern erweisen sich quantitative Methoden aus dem Bereich derjenigen Wissenschaften, die mit der theoretischen Einstufung des Lesebuchs als gesellschaftliche Institution der Kommunikation und Sozialisation angesprochen sind, als fruchtbar und legitim.

Die Interpretation der Befunde zeigt indessen die Notwendigkeit, die Grenzen dieser Methoden zu überdenken und Fragen zu stellen, die bisher unbeantwortet geblieben sind. Insbesondere mag es bedenklich stimmen, von „Außenlenkung” im 19. Jahrhundert zu sprechen, allenfalls noch von Leistungsorientierung. Inwieweit sich solche Aussagen überhaupt machen lassen und welchen konkreten Sinn sie im 19. Jahrhundert haben, läßt sich mit einer reinen Schulbuchanalyse nicht klären. Man müßte den Wandel der Sozialstruktur im Sinne einer Zunahme rollenspezifischer Beziehungen und leistungsorientierter Verhältnisse in den sozialen und wirtschaftlichen Bereichen direkt untersuchen, - durchaus eine Teilaufgabe auch des Generalthemas „Schulbildung und Industrialisierung". Doch auch eine Schulbuchanalyse bietet noch Möglichkeiten, das bisher gewonnene Bild zu ergänzen und zu vertiefen. Dokumentieren die Lesebuchgeschichten eine intendierte Verhaltenslehre, so muß es möglich sein, diese mit den Mitteln der Interpretation qualitativ zu bestimmen und ihre Funktion für die angesprochene soziale Schicht $z u$ beschreiben. Die Ergebnisse dieses Verfahrens dürften darüber hinaus Rückschlüsse erlauben zur Bewertung der quantitativen Befunde.

1 Siehe McClelland, Die Leistungsgesellschaft, Vorbemerkungen der Herausgeber (Ingeborg Y. Wendt), S. 12. 


\section{QUALITATIVE INHALTSANALYSE DER VERHALTENSLEHRE}

Die typische Lesebuchgeschichte des späten 18. Jahrhunderts exemplifiziert eine Tugend bzw. Untugend, z.B. „Der ehrliche Knabe”, „Das neugierige Kind" usw. Für die Zwecke dieser Untersuchung definieren wir „Tugend” mit „empfohlener Verhaltensweise”, wobei in der Regel ein System von Gratifikationen und Sanktionen mit der solcherart konstituierten Verhaltenslehre (Tugendkatalog) verbunden ist. Um welche „Tugenden" handelt es sich? Rochows Schilderung des Lebensweges Christians gibt uns ein Gliederungsprinzip für den Tugendkatalog nach dem Sprachgebrauch der Zeit in die Hand: ${ }^{1}$

„Das Glück des Tugendhaften schon hier auf Erden” "Christian war in der Jugend von seinen Eltern zur Schule gehalten und $\mathrm{zu}$ Fleiß und Rechtschaffenheit gewöhnt worden; daher war er verständig und liebte das Gute.

Als er groß wurde und heiraten wollte, da sah er vornehmlich nach einer fleißigen und tugendhaften Person, die er kannte. Daher war sein Ehestand glücklich; denn sie liebten sich beide und hielten über Ordnung und Zucht in ihrem Hause. Ihr beiderseitiger Fleiß machte denn auch, daß ihr Vermögen sich vermehrte, und von diesem Segen waren sie wohltätig und dienten gern mit Rat und Tat; daher war ihnen jeder gewogen. Sie gingen allem Zank aus dem Wege, mengten sich nicht in Dinge, die sie nichts angingen, und gaben jedem das Seinige. Daher blieben sie von Prozessen und Strafen frei, und die Herrschaft mochte sie ihrer guten Wirtschaft und Bescheidenheit wegen sehr wohl leiden. Weil sie mäßig lebten, sich nicht ärgerten und zankten, so blieben sie gesund und erreichten ein frohes Alter. Auch ihre Kinder gerieten wohl, weil sie ihnen mit gutem Beispiel vorgingen, und sie von Jugend auf gewöhnten, Gutes zu tun.

So ist die Tugend der sichere Weg zur Glückseligkeit."

„Fleiß” und „Rechtschaffenheit” sind es, die hier zur Quelle spezieller Tugenden werden und Glückseligkeit zur Folge haben. Sie sind erlernbar, wobei die Schule keine geringe Rolle spielt; sie sind nützlich, denn sie werden belohnt. Die volle Skala der zugeordneten Untertugenden entfaltet sich, wenn man alle Lesebuchgeschichten unter den Gesichtspunkten von „Flei $B$ ” und „Rechtschaffenheit” systematisch aufgliedert und zusammenstellt. Im folgenden sollen für 1780 und 1840 die Tugendsyndrome „Fleiß” und „Rechtschaffenheit” beleuchtet, ihr Verhältnis zueinander reflektiert und der Kontext aufgezeigt werden, der zu

1 Siehe Rochow, Der Kinderfreund..., (1776), Nr 58. 
ideologiekritischer Interpretation Anlaß bietet. Psychologisch gesehen, geht es um den "Charakter" des Rechtschaffenen, seine „Fähigkeiten” sowie seine „Motivationen”, „Strebungen”.

\section{0}

Zum „Charakter” des Rechtschaffenen gehören Ehrlichkeit, Ordentlichkeit und Mäßigkeit, wie eine Vielzahl von Geschichten illustriert. So hat der ,arme Schiffer", 1 ohne Sicherheiten bieten zu können, hundert Gulden auf seine „Redlichkeit” hin zu Kredit genommen. Die Zeit verstreicht; sollte er Treu und Glauben verraten haben? Nein, er kommt zurück, um Schulden zu tilgen und den Gewinn gleich mit abzuliefern. Solch Edelmut zwingt den Gläubiger, ihm großmütig die Schulden zu erlassen. Wird hier der Appell an Ehrlichkeit mit dem an Wohltätigkeit gekoppelt und bleibt die Geschichte wenigstens in dem Punkt realistisch, daß ein Kreditbetrug den Schiffer fernerhin kreditunwürdig machen würde, so können die Kinder aus den Illustrationen der Unehrlichkeit ${ }^{2}$ in ihren Formen des Lügens, Stehlens und Hehlens entnehmen, wie man es besser anstellen muB, um nicht entdeckt und bestraft zu werden.

Ordentlichkeit zeigt sich im Benehmen, in der Reinlichkeit, aber ebenso im Lebenswandel; Unordentlichkeit führt zu einem schlimmen Ende. Ludwig beschreibt einen „guten Hauswirt", ${ }^{3}$ der notwendige Reparaturen an seinem Haus sogleich ausführt, damit der Schaden sich nicht vergrößert; er duldet aus hygienischen Gründen keine „Wasseransammlung" nahe am Hause, verschafft sich nach Feierabend Bewegung durch Spaziergänge („,eine halbe Stunde”) oder Holzhacken (- die Scheite schichtet er ,ordentlich” hin - ); seine Wohnungen vermietet er preiswert, aber nur an "ordentliche Mietsleute”; sein gerechtes und höfliches Betragen läßt ihn weder mit Nachbarn noch mit der Obrigkeit Ärger haben; abends verschließt er selbst die Türen sorgfältig. „Bei seiner Mäßigkeit und Ordnung in allen Dingen erreichte er ein frohes Alter." Die „schlechten Mietsleute"' dagegen kehren ihre Stube nie aus, putzen keine Fenster, lassen ihre Kinder im Hause herumlaufen, werfen die Türen ins Schloß, hacken Holz in der Wohnung, zahlen keine Miete, sondern geben ihr Geld für „Branntwein, Kaffee und mancherlei Leckerbissen" aus, wobei sie sich an ihren zerrissenen Kleidern nicht stören. Das Ende vom Lied ist die Pfändung der Möbel, der Tod des Mannes „vom unmäßigen Branntweingenuß, der Blöße

1 Siehe Ludwig, Der Bürgerfreund ..., 7. Nützliche Erzählungen, Nr 18.

2 Vgl. a.a.O., Nr 3, 4; vgl. Rochow, Der Kinderfreund..., Nr 3, 7, 13, 47, 56.

3iehe Ludwig, Der Bürgerfreund..., 7. Nützliche Erzählungen, Nr 10.

4 Siehe a.a.O., Nr 11. 
und der schlechten Lebensart wegen". Die Frau muß mit ihren Kindern ums Brot betteln.

Ordentlichkeit koppelt sich hier mit Mäßigkeit. Eine Reihe von Geschichten wendet sich speziell gegen Luxus, meist in der Form, daB vor Verwöhnung gewarnt wird. ${ }^{1}$ Der Trunksucht widmet Rochow zwei Beispiele, wobei wiederum „Liederlichkeit” die Begleiterscheinung ist. ${ }^{2}$ Von Sparsamkeit ist in diesem Zusammenhang ganz selten die Rede, denn ein fleißiger und rechtschaffener Mann findet allemal sein Auskommen. Er kann freilich zu hoch greifen in seinen wirtschaftlichen Unternehmungen, wie der Bauer Georg, ${ }^{3}$ der mehrere Äcker kauft, dadurch in finanzielle Schwierigkeiten gerät und den Bauer Martin mit den Worten um Geld angeht: „,ihr habt wenig Land und könnt alles selbst aufs beste bestellen; ich aber muß teures Gesinde halten, und dieses arbeitet unwillig und träge, ackert schlecht, übertreibt mein Vieh zur Unzeit und ärgert mich krank. Dadurch bin ich zurückgekommen." „Wer auf einmal zu viel umfaßt, hebt nichts in die Höhe. Wer zu viel unternimmt, richtet wenig aus. Was ein arbeitsamer Mann selbst tut, gerät besser, als was er durch andere Leute tun läßt, die nur ums Brot arbeiten." Die Unmäßigkeit wird hier im wirtschaftlichen Bereich demonstriert, Rochows Morale koppelt den „Maßhalteappell” mit der Abwertung der Arbeitsleistung des Lohnarbeiters.

Die Arbeitsamkeit gehört dabei zu den zentralen Tüchtigkeiten eines Mannes vom „Charakter" der Rechtschaffenheit; Fleiß erst befähigt und macht nützlich. Dazu bedarf es des Lernens, und die Lesebücher werden nicht müde, diese Voraussetzung für jede nützliche Tätigkeit $\mathrm{zu}$ betonen, wobei die Autorität von Schule und Prediger stark unterstrichen wird, eine Haltung, die angesichts der noch nicht verwirklichten Schulpflicht nur zu verständlich ist. Ludwigs Johann ${ }^{4}$ wird von seinen Eltern so verwöhnt, daß er bei Regen oder Kälte die Schule nicht zu besuchen braucht. Er lernt „wenig Gutes”, in der Lehre ist ihm nichts recht, er wird eigensinnig und liederlich, schließlich stirbt er. Das „gute Schulkind” Karl dagegen ist reinlich und ordentlich, voller Aufmerksamkeit in der Schule, überdenkt zu Hause „das Gute, was er in der Schule gelernt hatte", wobei ihm der Vater hilft. In der Lehre gibt Karl gleichfalls acht auf alles, „was ihm sein Meister wies und sagte", und bringt es daher zu einem geschickten Gesellen. Man kann verstehen, wenn unter diesen Umständen das ,arme Kindermädchen”6

1 Vgl. a.a.O., Nr 6; Rochow, Der Kinderfreund..., Nr 49.

2 Vgl. Rochow, Der Kinderfreund..., Nr 34, 35.

Siehe a.a.O., Nr 43.

- Siehe Ludwig, Der Bürgerfreund..., 7. Nützliche Erzählungen, Nr 6.

s Siehe a.a.O., Nr 1.

- Siehe Rochow, Der Kinderfreund ..., Nr 4. 
nichts sehnlicher wünscht, als wie die anderen Kinder in die Schule gehen zu können: ,wenn ich daran gedenke, was aus mir werden wird, dann muß ich wohl weinen! Die andern Kinder gehen in die Schule und lernen viel Gutes, und ich wachse auf wie Unkraut. Ich habe nichts, um Schulgeld zu bezahlen; denn ich muß ums Brot dienen und bleibe also ungeschickt. Wer wird mich in Dienst nehmen wollen, wenn er geschicktere Leute bekommen kann? Ich wollte gern die Nacht arbeiten, wenn ich nur in die Schule gehen und was lernen dürfte!"

Die Nützlichkeit des Lernens exemplifiziert Rochow an Hans, ${ }^{1}$ der weder lesen noch schreiben kann und auf einen Betrüger hereinfällt, der ihm vorspiegelt, einen Schuldbrief auszustellen. Als Hans den Betrug merkt, sagt er: „Ach, hätte ich doch schreiben und lesen gelernt!” An seinen Kindern macht er nun gut, was er selbst versäumt hat. Fritz $^{2}$ weiß von dem Kantor, daß Apfelkerne, in die Erde gesät, wieder einen Baum hervorwachsen lassen, und er vollbringt mit seiner Schwester das Kunststück. Nach vielen Jahren, beim Äpfelpflücken, sagt Marie: „Wie gut war es, daß du in die Schule gingst und solche gute Sachen lerntest!” Rochow resümiert: „Ein guter Rat ist Geldes wert.” Wird hier der Wert des Lernens selbst zum Thema des Lernprozesses, so hat dieser noch andere Inhalte, die auf der Linie einer Indoktrination von Arbeitstugenden liegen und alle zum Komplex „Fleiß” gehören. Dem faulen Tagelöhner, ${ }^{3}$ der sich von seinem Kollegen sagen lassen muß, „nur den Faulen unter uns geht es schlecht. Wer arbeiten will und kann, dem mangelt nichts als das, was überflüssig und also entbehrlich ist", steht das mehrfach veranschaulichte Ideal treuer Pflichterfüllung gegenüber. Der "gute Knecht"4 nutzt die Krankheit seines Herrn nicht aus zu nachlässiger Arbeit, sondern arbeitet „doppelt fleißig". Der Bauer belohnt ihn mit Tochter und Hof. Ein Hirte, ${ }^{5}$ der mit Bibel und Gesangbuch ausgerüstet sein Vieh weidet, reflektiert: „Ich bin hier ganz allein; aber du, Herr, siehest und kennest mich! Nun will ich auch in meinem Amte treu sein, Schaden verhüten und Gutes tun. Denn das ist Gottes Wille. Und womit kann ich Armer meinen Dank gegen Gott besser beweisen als durch einen aufrichtigen Gehorsam?" So macht er sich an gemeinnützige Arbeiten wie Flußuferbereinigung, Zaunreparaturen, Kräutersuche. Als Belohnung findet er im Alter seine Versorgung im Dorf.

Pflichterfüllung als Gehorsam gegen Gott, als Gottesdienst in der Welt, diese Motivation läßt sich mehrfach belegen und motivgeschicht-

1 Siehe a.a.O., Nr 37.

${ }^{2}$ Siehe a.a.O., $\mathrm{Nr} 6$.

${ }^{3}$ Siehe a.a.O., $\mathrm{Nr} 41$.

4 Siehe a.a.O., Nr 23.

5 Siehe a.a.O., Nr 25. 
lich in ihrem Wandel zur Leistungsorientierung verfolgen. Als Beispiel diene das Thema von der ,guten Magd”. Rochow zeichnet sie 1776 zunächst folgendermaßen:1

„Christine diente bei einer schlimmen Herrschaft, die ihren Leuten wenig zu essen und beständig Scheltworte gab. Christine war arm, aber fromm. Sie betete oft zu Gott und sprach: ,Ach lieber Gott, lenke doch, wenn es dein guter Wille ist, das Herz meiner Brotherrschaft zu mir, daß sie mir nicht so hart und lieblos begegnet! Aber vielleicht ist mir diese Trübsal nützlich. Wer weiß, wie ich die guten Tage vertragen würde! Vielleicht würde ich frech und liederlich, wenn es mir zu wohl ginge. Du weißt es am besten, Herr, mein Gott! Schenke mir Geduld und hilf mir, daß ich treu und fleißig sei, wenn es mir gleich schlecht vergolten wird. Du, Herr, wirst alles wohlmachen und zu seiner Zeit mir Freude schenken."

Eine wohlhabende Witwe bemerkte Christines gute Aufführung, nahm sie zu sich und versetzte sie in gute Umstände.

Gott kennt der Freude rechte Stunde; Er weiß, wenn sie uns nützlich ist."

Hier sind Gottvertrauen und die reiche Witwe als deus ex machina die "Agenten” der Geschichte. Etwas weiter geht schon Ludwig 1787 mit der "fleißigen Magd”, deren Fleiß und Ordentlichkeit das Erstaunen der Nachbarin hervorruft. Marie antwortet darauf in traditioneller Bindung:2 „Gott, der alles sieht und weiß, hat mir meine gesunden Glieder nicht vergeblich, oder wohl gar zum Faulsein, sondern zur Arbeitsamkeit gegeben. Ich werde einmal davon Rechenschaft ablegen müssen, wozu ich meine Glieder und Kräfte gebraucht habe, und wie ich mit dem umgegangen bin, was mir anvertraut wurde."

Der Umbruch ist dann vollzogen in der letzten Ausgabe des Kinderfreundes zu Rochows Lebzeiten (1805) : $:^{3}$,Luise konnte spinnen, stricken, nähen, waschen, plätten, kochen und das Vieh füttern. Das alles hatte sie von ihren Eltern und anderen guten Leuten gelernt und abgesehen. Was sie davon konnte, das übte sie fleißig, so daß ihr alles recht leicht wurde. In der Schule hatte sie gut lesen, schreiben und rechnen gelernt. Dabei war sie reinlich, bescheiden, tat alles zur rechten Zeit und legte alles an den rechten Ort. Als sie groß wurde, bekam sie die beste Herrschaft, die sie wie ihr eigenes Kind hielt und liebte."

1 Siehe a.a.O., Nr 22.

'Siehe Ludwig, Der Bürgerfreund.... 7. Nützliche Erzählungen, Nr 7.

- Siehe Rochow, Der Kinderfreund..., (1805), in: Sämtliche Pädagogische Schriften, Band III, Berlin 1909, S. 355. 
Fleiß, Arbeitsamkeit, Lernen nützlicher Fertigkeiten und ihre Anwendung, Pflichterfülllung, diese Tugenden führen zu Erfolg, ob dieser nun aus der Erfüllung göttlich gebotener Berufsauffassung hervorgeht oder zum Ergebnis von Leistungen in dem Spiel von Angebot und Nachfrage säkularisiert ist. Der Zusammenhang von „Rechtschaffenheit” und „Fleiß”, von „Charakter” und „Fähigkeiten” in der Verhaltenslehre, wie sie die Lesebuchgeschichten von 7780 bieten, wird damit deutlich. Das Akzeptieren einer Moral im Sinne von Ehrlichkeit, Ordentlichkeit und Mäßigkeit bildet die psychologische Voraussetzung, die das Interesse an jedem Lernen, an der Schule selbst, an beruflichen Fertigkeiten wecken mußte; sie erst konnte empfindlich machen für Erfolg bzw. Mißerfolg, für Gratifikation und Sanktion, und damit Fleiß, Arbeitseifer, Pflichterfüllung und Gehorsam stimulieren. Nur ein Arbeiter, der etwas auf sich hielt, bot die Chance, willig und eifrig die spezifischen Arbeitstugenden $\mathrm{zu}$ praktizieren. ${ }^{1}$ Insofern ließe sich sagen, daß die Tugenden der „Rechtschaffenheit”, wie wir sie vorgestellt haben, nicht eigentlich "Charakter" konstituieren, sondern weitgehend instrumental $\mathrm{zu}$ verstehen sind und ebenso wie „Fleiß” mit seinen Spielarten Verhaltensweisen der Soziabilität darstellen, die den Menschen zum „nützlichen Glied der Gesellschaft” qualifizieren. So sehr dabei „Rechtschaffenheit” und „Fleiß” gesamtgesellschaftliche Desiderate eines gedeihlichen Zusammenlebens sein mögen, so kann man ihre Propagierung doch auch ideologiekritisch sehen. Denn die „Nützlichkeit” der Tugenden für den praktizierenden Menschen wird in Form einer Belohnung zwar behauptet, die Funktion dieser Nützlichkeit für den Nutznießer, in der Regel die „Herrschaft”, aber nicht mehr thematisiert. Der Tugendhafte dient scheinbar letztlich nur sich selbst, wenn er tugendhaft ist: er schützt sich vor Strafe.

Wird damit die Abhängigkeit des Menschen von einer Autorität gesetzt, die ihm sein Verhalten vorschreibt mit der lockenden Aussicht auf Belohnung, so verstärkt der Kontext diese Abhängigkeit. Denn hier werden den Verhaltensweisen der handelnden Personen in den Lesebuchgeschichten Motivationen bzw. Strebungen unterlegt, die einseitig Selbsthingabe beinhalten: Liebe der Kinder zu den Eltern, Wohltätigkeit gegenüber dem Armen und Hilfsbedürftigen, Vertrauen in Gottes Allmacht, Weisheit, Güte und Hilfe. Von Strebungen der Selbstbehauptung, von Egoismus, Ehrgeiz, Herrschsucht, Erwerbssinn ist keine Rede. Die Zahl der Lesebuchgeschichten zu den Themen Wohltätigkeit und Gottvertrauen ist beträchtlich, wobei auch hier die

1 Vg1. Pollard, Factory Discipline in the Industrial Revolution, S. 267ff. ; ebenso: The Genesis of Modern Management. A Study of the Industrial Revolution in Great Britain, London 1965, S. 193. 
Belohnungen nicht ausbleiben. Zwei Beispiele mögen genügen: Die vertriebenen Fremden, ${ }^{1}$ von einem gastfreien Dorf aufgenommen, lehren „aus Dankbarkeit die Leute im Dorfe viele neue und nützliche Dinge und verschiedene Handgriffe", wodurch sich der Wohlstand des Dorfes mehrt. Und dem „wohltätigen Kind", ${ }^{2}$ das sein Brot mit einem Bettler teilt, geht es „wohl”, als es groß geworden ist. Gottvertrauen konnte bereits an der guten Magd Christine (in der Fassung von 1776) gezeigt werden.

Um nicht mißverstanden $z u$ werden: Wohltätigkeit, Liebe und Gottvertrauen sollen in ihrem Wert nicht zur Diskussion gestellt werden. Hier geht es darum, daß eine einseitige Betonung von Werten der Selbsthingabe den Ideologiegehalt der Lesebücher im Sinne einer Ausrichtung auf Einordnung in Herrschaftsverhältnisse verstärkt. Mit einer einzigen Geschichte scheint Rochow diesem Bilde $\mathrm{zu}$ widersprechen und autonomem Erwerbssinn das Wort zu reden:3 „Der kluge Wirt bei der Teurung”: „Als einst bei nasser Witterung das Getreide schlecht geraten war, und der Scheffel Roggen drei Taler galt, da rechnete Georg aus, daß er sonst gewöhnlich sechzig Scheffel Roggen zu Brot gebraucht hätte. Er fing also gleich nach der Ernte zu sparen an und kaufte drei Wispel Erdtoffeln, für sechzehn Taler den Wispel, das waren achtundvierzig Taler. Und nun verkaufte er dreißig Scheffel Roggen für neunzig Taler, weil er statt des mehreren Brots nun Erdtoffeln speisete, und gewann auf diese Weise bei der teuren Zeit, da fast ein jeder verlor, zweiundvierzig Taler." Aber das Morale fälscht den kapitalistischen Kalkül um in die Sorge des guten Hausvaters, der es nicht nötig hat, in einer Notzeit zu sparen, weil er beizeiten vorgesorgt hat: „Denke in Zeiten daran, wie du dich einrichten willst; denn wenn die Not einbricht, so ist es zu spät."

Zusammenfassend läßt sich für 1780 sagen: Rochow und Ludwig, so unterschiedlich ihre Lesebücher auch sonst sind, zeichnen mit großer Übereinstimmung in der Verhaltenslehre eine statische Welt, in der der Tugendhafte seinen Platz hat, wenn er sich durch Rechtschaffenheit und Fleiß qualifiziert, wobei er sich durch Gottes Willen bestimmt und im Vertrauen zu Gott gehalten weiß. Der Status quo erhält die Weihe der Theodizee: „Es ist mehr Gutes als Böses in der Welt", überschreibt Rochow die Geschichte und läßt den Vater gegenüber seinen Kindern ausführen: „Das Unangenehme in dem menschlichen Leben ist entweder verschuldet, und dann ist es Strafe, zur Besserung nützlich, oder es trifft uns, ohne daß wir es veranlaßt

I Siehe Rochow, Der Kinderfreund..., Nr 40.

${ }^{2}$ Siehe Rochow, a.a.O., Nr 26.

Siehe a.a.O., Nr 57.

- Siehe Rochow, a.a.O., Nr 62. 
haben, und dann ist es Schickung oder Verhängnis des allerweisesten Gottes und Vaters und im ganzen gewiß gut und nützlich." So gut die Welt auch eingerichtet ist, „, beständige Glückseligkeit ist nach dem Tode der Lohn des Frommen", tröstet Rochow den Zukurzgekommenen.

\section{0}

Die Welt von 1780 blieb trotz der „Nützlichkeit” ihres Status quo vor tiefgreifenden politischen, sozialen und ökonomischen Umwälzungen nicht verschont. Wie spiegelt sich eine veränderte Welt in den Lesebüchern von 1840, welche Unterschiede im Bereich der Verhaltenslehre zeigen sich zwischen 1780 und 1840, wo liegt Veranlassung zu ideologiekritischer Bewertung der Befunde?

Es überrascht nicht, daß „FleiB” und „Rechtschaffenheit” wesentliche Bestandteile des Verhaltensmusters bleiben. Der "arme Schiffer” aus Ludwigs Bürgerfreund, ${ }^{1}$ der auf Treu und Glauben Kredit bekommt und ihn ehrlich zurückzahlen will, findet sich wörtlich im Berlinischen Lesebuch von Schulz (1840). ${ }^{2}$ - Die Ehrlichkeit des Wandersmannes, der, von einem Räuber überfallen, diesem verspricht, sein Geld aus der Stadt zu holen und brüderlich zu teilen, rührt den Räuber zu großmütigem Verzicht. - Unmäßigkeit macht unvernünftig, wie Schulz an zwei Beispielen demonstriert, ${ }^{3}$ Ordentlichkeit wird dagegen nicht mehr Thema der Verhaltenslehre. Uberhaupt scheint die Notwendigkeit, diese Art „Rechtschaffenheit” zu betonen, geringer geworden zu sein. Was die Tüchtigkeiten unter dem Stichwort „Fleiß” betrifft, so stellen beide Lesebücher Arbeitsamkeit als Quelle des wirtschaftlichen Erfolges vor. Bei Schulz fordert der sterbende Vater seine Söhne auf, nach dem im Weinberg verborgenen Schatz zu graben. Doch alle Mühe ist vergebens, kein Schatz ist zu finden, bis im folgenden Jahr die Weinstöcke dreifache Frucht tragen. ${ }^{4}$ Ähnlich korrigiert im Preußischen Kinderfreund der Reiche das Bemühen seines armen Nachbarn, aus dem Boden einen Schatz zu Tage zu fördern, indem er ihm einen kleinen Obstbaum für das gegrabene Loch schenkt, der vielfältige Frucht trägt. Der arme Nachbar merkt sich den „Leibspruch des klugen Nachbarn”: „Ein sichrer Reichtum ist Verstand / Und eine arbeitsame Hand."5

Die Forderung, Arbeit voran- und seine privaten Freuden zurückzustellen, steht drohend hinter den abschreckenden Beispielen, wenn

1 Vgl. S. 104, Anm. 1.

2 Siehe Schulz, Berlinisches Lesebuch..., IV, Nr 2.

3gl, a.a.O., IV, Nr 24; V, Nr 1.

4 Siehe a.a.O., Nr 13.

5 Siehe Preuß/Vetter, Preußischer Kinderfreund, III, Nr 122. 
solches nicht geschieht. Franz ${ }^{1}$ läßt sich vom schönen Wetter verführen, die aufgetragene Arbeit zu verschieben. Doch das Versäumte läßt sich nicht nachholen, jahreszeitliche Unbill macht jeden Versuch zuschanden. Schulz resümiert: „Was du tust, das tue bald. Jeden Aufschub heilsamer Geschäfte wirst du bereuen." Hier spricht sich bürgerliches Arbeitsethos aus, freilich theologisch interpretiert mit dem Nachsatz: „Wie beschämt wirst du im Alter einst sein, wenn du den schöneren Teil deines Lebens hast verstreichen lassen, ohne ihn im Dienste Gottes zu nützen." Daß dieses Arbeitsethos nicht Mittel für Erwerbsstreben ist, wird indessen sofort deutlich, wenn man den Kontext berücksichtigt. Hier ist zunächst nur festzustellen, daß Arbeitsamkeit einen zentralen Platz im Bereich der nützlichen Tüchtigkeiten einnimmt. Schulz, der gegenüber Preuß/Vetter den Fächer der Tüchtigkeiten stärker differenziert, gibt einige Beispiele für bislang nicht erwähnte Tugenden aus diesem Bereich, so für Klugheit,, ${ }^{2}$ Genauigkeit, ${ }^{3}$ Sparsamkeit ${ }^{4}$ und Strenge, ${ }^{5}$ wobei Sparsamkeit als Mittel gesehen wird, den Eltern ihre Liebe durch Unterstützung im Alter zu vergelten, eine Deutung, die erneut auf den Kontext verweist.

Der Kontext nimmt 1840 einen viel breiteren Raum ein als 1780 , die Strebungen und Einstellungen des Menschen werden stärker differenziert, freilich ebenso einseitig ausgewählt und bewertet. Mit der besonders bei Preuß/Vetter verstärkten Betonung der Wohltätigkeit werden zunächst erneut Strebungen der Selbsthingabe mobilisiert, ${ }^{6}$ hier nun in auffälliger Weise verbunden mit dem Motiv der von außen kommenden Hilfe, die nicht rational zu planen und vernünftig zu kalkulieren ist, sondern dem karitativen Eingriff Gottes, des Landesherrn oder einer übergeordneten Person entspringt. Die „Almosengeschichte" wird damit zum "Zuckerbrot", das neben der „Peitsche” einer rigorosen Arbeitsmoral liegt.

So trifft der ,kleine Börsenhändler"7 bei seinem Versuch, wenigstens das Abendbrot für diesen Tag zu verdienen, auf den König, der mit seiner Tochter im Tiergarten spazierengeht. Die Schilderung der Not bewegt den König, einige gestickte Börsen zu einem freiwilligen Überpreis abzukaufen, ja er kümmert sich um den Jungen und setzt der

1 Siehe Schulz, Berlinisches Lesebuch..., V, Nr 15 und bei Preuß/Vetter, Preußischer Kinderfreund, III, Nr 179.

${ }^{2}$ Vgl. Schulz, Berlinisches Lesebuch..., IV, Nr 15.

3 Vgl. a.a.O., IV, $\mathrm{Nr} 17$.

Vgl. a.a.O., V, Nr 16.

5 Vgl, a.a.O., V, Nr 25.

- Vgl, z.B. PreuB/Vetter, Preußischer Kinderfreund, III, Nr 213.

7 Siehe a.a.O., III, Nr 142. 
Soldatenwitwe ein Jahrgeld aus. - Einen gefährlichen Weg, um zu Geld zu kommen, schlägt ein Familienvater ein, ${ }^{1}$ der seine verhungernde Familie durch mit vorgehaltener Pistole erpreßtes Geld zu ernähren sucht. Auch hier forscht der unfreiwillige Spender der Sache nach, überzeugt sich von der unverschuldeten Armut und verzeiht mit den Worten: „Ich weiß, ihr seid ein Schuster. Hier habt ihr dreißig Goldstücke, die schenke ich euch, kauft Leder dafür und verdient von nun an durch Arbeit, was ihr für euch und für eure Kinder braucht."

Bleibt der Reiche aber hartherzig und läßt sich von der unverschuldeten Armut nicht beeindrucken, so nimmt er ein schlimmes Ende, wie der Hausbesitzer Pohl, ${ }^{2}$ der eine Witwe mit ihren Kindern vor die Tür setzt, weil die Mutter wegen der Pflege eines kranken Kindes an der Arbeit gehindert und damit in Mietschulden geraten war. Das schlechte Gewissen bringt den Hartherzigen zu Krankheit und Tod. Hier wird bereits die Thematik "Reichtum und Tugend" berührt. Dem Wohltäter steht in gleicher Breite der durch Reichtum Verdorbene gegenüber. Ist dieser der Verführung des Reichtums erlegen, so gilt es für den einfachen Mann, dieser Verführung zu widerstehen und tugendhaft zu bleiben. Schulz, dessen Berlinisches Lesebuch dieses Thema stärker repräsentiert als Preuß/Vetter, gibt den Prototyp: Der arme Amynt $^{3}$ kann nur unter großen Demütigungen den reichen Arbeitgeber dazu bewegen, ihm einen kleinen VorschuB zu geben. Als in dieser Situation ein Handwerker alte Forderungen an den Reichen geltend macht, sich abgewiesen und beschimpft sieht und ihn vor Gericht bringen will mit Amynt als Zeugen, versucht der Reiche, Amynt zu bestechen. Doch Amynt bleibt standhaft: „ich habe seit zween Tagen / für meine Kinder nicht satt Brot; / sie werden über Hunger klagen, / sobald sie mich nur wieder sehen:/ es wird mir an die Seele gehn;/ die Schulden werden mich aus meiner Hütte jagen; / allein ich will's mit Gott ertragen. / Streicht euer Geld, das ihr mir bietet, ein, / und lernt von mir die Pflicht, gewissenhaft zu sein."

$\mathrm{Da} B$ man mit Geld nicht alles kaufen kann, muß auch ein reicher Kaufherr ${ }^{4}$ erfahren, der sich durch das ständige fröhliche Singen seines armen Nachbarn gestört fühlt und sich die Ruhe erkaufen will. Der Handwerksmann geht zunächst lachend darauf ein: „Als er mit dem Gelde nach Hause gekommen, überzählte er es voller Freuden, und es war lauter gute Münze, und so viel, als er noch niemals beisammen hatte. Abends, ehe er schlafen ging, liebäugelte er noch ein

1 Siehe a.a.O., III, Nr 147.

2 Siehe a.a.O., III, Nr 120 und bei Schulz, Berlinisches Lesebuch..., V, Nr 12.

${ }^{3}$ Siehe Schulz, Berlinisches Lesebuch..., IV, Nr 3.

' Siehe a.a.O., V, Nr 24.; die gleiche Geschichte in Versen: a.a.O., IV, Nr 27. 
gutes Stündchen mit seinem Schatze, und Nachts legte er es unter sein Kissen, damit es ihm nicht etwa ein Dieb rauben könnte; und um Mitternacht hatte er es noch im Kopfe, und sann nach, was er damit anfangen und wie viel er gewinnen könne an Kapital und Zinsen; und Morgens, wie er aufstand, lag es ihm in allen Gliedern wie Blei; sein Kopf war wüst von Nachtwachen und Sorgen, seine Hand schwer und lässig, und versagte ihm den Dienst, und er durfte nicht singen." Das ist zuviel für den armen Handwerksmann. Er nimmt das Geld und eilt zu seinem Nachbarn: „Herr, mit Vergunst,... da habt ihr euren Plunder wieder; der Kobold läßt mich nicht schlafen... Ein frischer, froher Mut geht über Geld und Gut. Trilirum, tralarum!"

Die Dämonisierung des Reichtums, wie sie diese Geschichte zeigt, steht in innerem Zusammenhang mit dem auch wieder bei Schulz besonders stark vertretenen Thema der Zufriedenheit. Schon Ludwig widmet 1787 eine Nummer seiner „nützlichen Erzählungen” diesem Thema; er bietet ein Gespräch zwischen „Arbeitsleuten” an:1

„Hans: ..., wenn man bei schwerer Arbeit zu nichts kommen, sich nichts zu Gute tun kann, und doch sehen muB, daB mancher bei weniger Arbeit viel Geld verdient... ; so muß man wohl verdrüßlich werden.

Karl: Der Vornehme, der viel Geld verdient, braucht auch mehr als wir... Wahre Glückseligkeit besteht gar nicht in Reichtum und vornehmen Stande. -

[Gibt ein Beispiel, wie er einmal eine Stelle gehabt habe, wo nur gegessen und kaum gearbeitet wurde; schließlich sei er krank geworden, erst wieder bei der gewohnten Arbeit und Kost genesen.] Gott hat uns nach seiner großen Weisheit und Güte von Eltern mittelmäßigen Standes geboren werden lassen, und uns in diesen Stand, der gewiß der beste für uns ist, gesetzt...

Hans: ...Wenn unsere Arbeit aber nur besser bezahlt würde!

Karl: Sie wird doch so bezahlt, daß man das Nötige für den Verdienst anschaffen kann; um das Entbehrliche werde ich mich nie grämen, sondern Gott stets für das Meinige danken und dabei zufrieden sein. Ich werde das nicht so hoch achten und mir das wünschen, was ich nicht habe. Mir fällt eben ein schöner Vers ein, den ich von meinem Lehrer in der Schule gelernt habe, und der mich oft recht zufrieden mit meinem Stande gemacht hat; er heißt so:

Genieße, was dir Gott beschieden, / entbehre froh, was du nicht hast, / wie jeder Stand hat seinen Frieden / so hat auch jeder seine Last.

Durch Murren wächst nur unser Leiden, / führwahr es mindert

1 Siehe Ludwig, Der Bürgerfreund..., 7. Nützliche Erzählungen, Nr 22. 
keine Not, / sei froh und danke Gott mit Freuden / für Wasser und für Salz und Brot."

Entspricht Karls Auffassung dem statischen Weltbild, das wir für die Schulbücher von 1780 insgesamt charakteristisch fanden, ein Weltbild, in dem in der Tat „Murren” als einzige Alternative möglich erscheint, so ist der Ideologiegehalt in der Behandlung des Themas "Zufriedenheit" bei Schulz 1840 ungleich deutlicher und allein schon an der Breite des ihm zugemessenen Raumes ablesbar. „Nicht zu hoch hinaus", das ist das Thema von zwei Fabeln: Der Fuchs ${ }^{1}$ verzichtet auf die unerreichbaren Weintrauben und tröstet sich: „Was soll ich mir viel Mühe nehmen? Sie sind ja herb und taugen nichts." Der Hänfling $^{2}$ baut sein Nest erst in der Eiche, die dem Blitz ausgesetzt ist, dann auf der Erde, wo das Vieh das Nest zertritt, worauf er eine Mittellage wählt, die ihm Ruhe gewährt. Denn: „Vergnügte Tage findet man, / wenn man sie hier noch finden kann, / nicht bei dem Thron, nicht in den Hütten. / Kannst du vom Himmel es erbitten, / so sei dein eigener Herr und Knecht. / Dies bleibt des Mittelstandes Recht."

Vertikale Mobilität ist nicht gut für jedermann, will Schulz sagen, wenn er einen guten Karrenschieber ${ }^{3}$ nach seiner Beförderung zum Kutscher scheitern läßt. - Die ,goldne Zeit"4 hat es dem Bauernsohn angetan, das Leben im Schlaraffenland. Nein, sagt der Vater, dann ginge es uns schlechter, weil der Junker sich alle Früchte anmaßen würde und keine Arbeit mehr zu vergeben hätte. Auf des Sohnes schüchternen Einwand, Gleichheit unter den Menschen gehöre zur goldnen Zeit, antwortet der Vater: „Noch besser! Alle gleich! Ei, was für Zanken, Streiten / und Morden möchte da nicht oft entstehn! / Wie oft nicht da Gewalt für Recht ergehn!/ Nein, jetzt kann Jeder doch, was er erwirbt, behalten; / hat ruhig sein Stück Brot, das Arbeit ihm versüßt. / Drum geh mit deiner goldnen Zeit der Alten / und $\mathrm{la} B$ die Welt so, wie sie ist!" Status quo - Ideologie begreift sich hier als fortschrittlich, die Alternative siedelt im literarischen Topos vom goldnen Zeitalter.

Bildungsbürgerliche Kompensation für die vom Dämon Reichtum bedrohte Tugend wird nahegelegt, wenn man sieht, daß die Thematik der Zufriedenheit auch in die Poesie, in den vom Lesebuch dem „Schönen" eingeräumten Bereich Eingang findet. Die zwei Vögel ${ }^{5}$ Habich

1 Siehe Schulz, Berlinisches Lesebuch..., IV, Nr 22.

Siehe a.a.O., IV, Nr 23.

Siehe a.a.O., IV, Nr 21.

1 Siehe a.a.O., IV, Nr 19.

- Siehe a.a.O., VII, Nr 24. 
und Hättich verschaffen dem Menschen Ruhe, Zufriedenheit oder Unrast, Unzufriedenheit: „Drum läßt sich jeder verständige Mann / an seinem Habich genügen, / und lacht ihn auch manchmal ein Hättich an, / so läßt er mit Gleichmut ihn fliegen.” Mit dem „Habich” in der Hand soll der Mensch „,täglich singen": 1

„Ich danke Gott mit Saitenspiel, / daß ich kein König worden; / ich wär' geschmeichelt worden viel, / und wär' vielleicht verdorben. /

Auch bet' ich ihn von Herzen an, / daß ich auf dieser Erde / nicht bin ein großer, reicher Mann, / und auch wohl keiner werde. Denn Ehr' und Reichtum treibt und bläht, / hat mancherlei Gefahren, / und Vielen hat's das Herz verdreht, / die weiland wacker waren. /

Und all' das Geld und all' das Gut / gewährt zwar viele Sachen; / Gesundheit, Schlaf und guten Mut/kann's aber doch nicht machen. /

Und die sind doch, bei Ja und Nein! / ein rechter Lohn und Segen:/ drum will ich mich nicht viel kastei'n / des vielen Geldes wegen. /

Gott gebe mir nur jeden Tag, / was ich bedarf zum Leben; / er giebt's dem Sperling auf dem Dach, / wie sollt' er's mir nicht geben?"

Das Deutsche Lesebuch Philipp Wackernagels, vorwiegend an höheren Schulen im Gebrauch und der Kategorie des „literarischen Lesebuchs" zugehörig, ${ }^{2}$ zeigt die bildungsbürgerliche Thematisierung von "Reichtum" und „Zufriedenheit” in letzter Ausformung: Der Schatzmeister ${ }^{3}$ des Königs, vom Hirten in dieses Amt avanciert, wird der Veruntreuung bezichtigt, weil er einen mit einer eisernen Tür verschlossenen, geheimnisvollen Raum täglich betritt. Der König überprüft die Anschuldigung und entdeckt

„vier leere Wände, einen ländlichen Tisch und einen Strohsessel. Auf dem Tische lag eine Hirtenflöte, nebst einem Hirtenstabe und einer Hirtentasche. Durch das Fenster sah man auf grüne Wiesen und waldige Berge.

Der Schatzmeister aber sprach: ,In meiner Jugend hütete ich die Schafe. Du, o König, zogst mich an deinen Hof. Hier in diesem Gewölbe brachte ich nun täglich eine Stunde zu, erinnerte mich mit

1 Siehe a.a.O., VII, Nr 25 und I, Nr 12.

2 Vgl. Roeder, Geschichte und Kritik des Lesebuchs..., S. $188 \mathrm{ff}$.

3 Wackernagel, Deutsches Lesebuch, I. Teil, Nr 32. 
Freuden meines vorigen Standes, und wiederholte die Lieder, die ich ehemals bei meinen Schafen zum Lobe des Schöpfers gesungen hatte. Ach laß mich wieder zurückkehren auf meine väterlichen Fluren, wo ich glücklicher war, als an deinem Hofe!'

Der König ward über die Verläumder sehr unwillig, umarmte den edlen Mann und bat ihn, ferner in seinen Diensten zu bleiben.

Ein ruhiges Herz, nicht Gold und Pracht, ist's, was uns Menschen glücklich macht."

Der geheimnisvolle Raum mit der eisernen Tür ist die Innerlichkeit des tugendhaften Menschen. In diesem Raum findet Entlastung und Kompensation, wer in der Welt den Gefahren des schnöden Mammons ausgesetzt ist. Wem diese Gefahren gar nicht erst drohen, der kann sich an gleicher Stelle trösten lassen über die wahren Werte. Beide verlassen „zufrieden” diesen Raum, um sich an die Arbeit zu begeben.

Versucht man, die Ergebnisse der qualitativen Inhaltsanalyse für 1780 und 1840 miteinander zu vergleichen und die Befunde zusammenfassend $\mathrm{zu}$ interpretieren, so läßt sich folgendes sagen: Rochow und Ludwig räumen 1780 den Tugendsyndromen "Rechtschaffenheit” und „Fleiß” einen breiten Raum ein, um den Menschen für den Arbeitsprozeß allererst zu qualifizieren. Preuß/Vetter und Schulz halten 1840 daran fest, verstärken die Betonung der Wohltätigkeit, bereichern dies Thema um die Almosengeschichte und gesellen als neue Themen die Dämonisierung des Reichtums und das Lob der Zufriedenheit hinzu. Fragt man nach der Funktion dieser Veränderungen, so liegt folgender SchluB nahe: LieB sich der Arbeiter in seiner Rolle qualifizieren, partizipierte er an den „bürgerlichen” Werten des Fleißes und der Rechtschaffenheit, steigerte er sein "Leistungsbedürfnis”, dann bestand die Möglichkeit, daß er die Chancen einer im Sinne der Leistungsorientierung veränderten Sozialstruktur wahrnahm und unternehmerisches Rollenverhalten entwickelte. Hier lag ein "Dilemma”" wie konnte man den Arbeiter qualifizieren, ohne ihn damit zu verlieren? Die Unterbewertung kapitalistischer Erfolge, die Idealisierung einer unterprivilegierten Lebensform, das Insistieren auf inneren Werten, diese Methoden mußten, ob bewußt eingesetzt oder subjektiv ehrlich als Wert begriffen, jenen antiemanzipatorischen Effekt haben, der das Dilemma im Sinne der bestehenden Herrschaftsverhältnisse löste.

Schulz bietet eine einzige Geschichte, die diesem Bild eine kleine

1 Vgl. Pollard, Factory Discipline in the Industrial Revolution, S. 270f.; vgl. auch Friedrich Naumann, Volksschule und industrielle Entwicklung, Festvortrag, Berlin o.J., S. 14: „Die Frage, ob im Gesinnungsunterricht mehr die passiven oder mehr die aktiven Tugenden zu pflegen seien, ist das eigentliche Kampfgebiet." 
Korrektur zu geben scheint: Dionys, ${ }^{1}$ Tyrann von Syrakus, raubt einem Reichen dessen vergrabenen Schatz. Der Reiche fängt von vorne an, treibt Landwirtschaft und kommt wieder zu Vermögen. Dionys ruft ihn zu sich und belehrt den vor Furcht Zitternden. „Ich habe dir beweisen wollen, / daß, wenn das Glück uns günstig ist, / wir's nützen, nicht verscharren sollen. / Besitze nun dein Gut in Ruh; / hier ist der Schatz, den du vergraben / und den ich dir geraubt; nimm diesen auch dazu. / Jetzt bist du würdig, ihn zu haben." Die Aufforderung, mit seinem Pfund zu wuchern, ergeht an den Reichen, der ohnehin den „Beruf” hat, reich zu werden, wie sein Schicksal zeigt. Er soll also nicht zufrieden sein mit dem Erreichten, seine „Nützlichkeit" für den Staat besteht in der Unternehmung. Das antike Vorbild mag dem Unternehmer von 1840 Selbstrechtfertigung seines Tuns bedeutet haben, für den Arbeiter und kleinen Handwerker konnte daraus kaum ein Stimulans erwachsen, die im Kontext der Lesebuchgeschichten gesetzten Grenzen der indoktrinierten Verhaltensweisen zu überschreiten.

\section{ZUSAMMENFASSUNG UND AUSBLICK}

Die Ausgangsfrage dieser Untersuchung war, ob sich mit Schulbüchern aus der Zeit der Frühindustrialisierung ein Umschlagplatz zur gesamtgesellschaftlichen Durchsetzung des „kapitalistischen Geistes” bzw. eines „bürgerlichen Wertsystems” ausmachen läßt oder welche sozioökonomisch relevanten Verhaltensnormen indoktriniert werden. Die Ergebnisse der mit verschiedenen Methoden vorgenommen Inhaltsanalyse zeigen, da $B$ etwa der Franklinsche Tugendkatalog ${ }^{2}$ als Ausdruck des bürgerlichen Tugendideals nicht in Widerspruch steht zu der propagierten Verhaltenslehre, daß aber charakteristische Zusätze, wie „Pflichterfüllung” (7780) und „Zufriedenheit”, „Dämonisierung des Reichtums" (1840) den allgemeinen Trend schichtenspezifisch modifizieren im Sinne der "Schaffung” einer den „Bedürfnissen” des „gemeinen Mannes" angepaßten Bildung. ${ }^{3}$ Der „kapitalistische Geist” Max Webers wird in dem Sinne indoktriniert, daß der Arbeiter die Arbeit als Beruf begreift, nicht aber den Erwerb. ${ }^{4}$ Die allgemeine

1 Schulz, Berlinisches Lesebuch..., IV, Nr 14.

2 Siehe Benjamin Franklin, Autobiographie, Übersetzung von Berthold Auerbach, durchgesehen von Rolf Dornbacher, Frankfurt/M., 1969, S. $126 f$.

${ }^{3}$ Ebenso: Pollard, Die Bildung und Ausbildung der industriellen Klassen..., S. 27.

4 Vgl. Max Weber, Die protestantische Ethik, S. 186; in Sprache und Selbstverständnis eines Unternehmers der Zeit heißt die Dichotomie „fleißige Hände” und ,unternehmende Geister”, vgl. Hartmut Kaelble, Die Berliner Unternehmer, Herkunft, sozialer Status und politischer Einfluß 1835-1873, Manuskript, Berlin 1970, S. 86. Kaelble zitiert H. C. Carl, Deutschland's Zolleinigung. Schutz- und Differentialzölle, Frankfurt/M. 1848, S. 31. 
Entwicklung spiegelt sich, sofern mit diesen Methoden meßbar, in dem Ansteigen des Niveaus an „Leistungsbedürfnis” und der stärkeren Einstellung auf „Außenlenkung”. Die schichtenspezifische Ausrichtung verläuft als Bremswirkung oder Störeffekt, sie kanalisiert Leistung und Außenlenkung in die Erfordernisse der „arbeitnehmerischen Rolle” (und gesamtgesellschaftlich in die Untertanen-Rolle) und tröstet mit der Ideologie von Zufriedenheit und Verpönung des Reichtums. Die Lesebücher erweisen sich damit gerade durch ihre scheinbar wirtschaftsfremde Attitüde als wirtschafts- und sozialpolitisch außerordentlich parteiisch.

Man kann einwenden, daß hier lediglich untersucht wurde, welches Bild der Welt und eines weltgerechten Verhaltens vier Autoren 1780 und 1840 entworfen haben, womit noch nichts ausgesagt sei über die Schulwirklichkeit, den Grad der Beeinflussung der Schüler durch die Lesebücher, über die Repräsentanz dieser Lesebücher gegenüber anderen oder der Schulbücher überhaupt für einen wie immer gearteten „Geist der Zeit”. Die Schulwirklichkeit kann zweilfellos durch Schulbuchanalyse nicht erfaßt werden, und die Breite des zugrundegelegten Quellenmaterials ist zugegebenermaßen klein. Der „cultural lag” zwischen dem angeblichen "Geist der Zeit" und den Schulbüchern mag bestehen, ist jedoch seinerseits Signum dieses Zeitgeistes. Jedenfalls gewinnt das Bild, das sich aus den wenigen Lesebüchern ergibt, an innerer Wahrscheinlichkeit und Glaubwürdigkeit, weil es in den Rahmen des deutschen bzw. preußischen Bildungswesens des 19. Jahrhunderts paßt, aber auch jenen Zuständen entspricht, die Pollard für das 18. und frühe 19. Jahrhundert in Großbritannien kennzeichnet. ${ }^{1}$

Wenn in Preußen der Staat das Realschulwesen ebenso wie das technische Hoch- und Fachschulwesen fortlaufend ausbaut, bedient er damit zugleich ein wachsendes Bedürfnis des Wirtschaftsbürgertums nach Qualifizierung durch Kenntnisvermittlung und legt den Grundstein zu der technologischen Führungsrolle Deutschlands in Europa am Ende des Jahrhunderts. Durch das rigorose Berechtigungswesen jedoch, das an bestimmte Lateinkenntnisse gebunden war und damit die höheren Schulen einseitig privilegierte, die Volksschule aber isolierte, schließt sich dieses Bildungswesen zunehmend sozial nach unten ab. Die Institution "Schule" gewinnt in der Klassengesellschaft, deren Prinzipien im Zeitalter des Kapitalismus Leistungsorientierung und Rollenspezifizierung sein sollten, noch nicht oder nur

1 Pollard veranschlagt die Bedeutung des Schulwesens für das frühe Management relativ hoch, für die Arbeiterschaft weniger wichtig, aber auf die Arbeitsmoral konzentriert. Vgl. The Genesis of Modern Management, S. 104ff. und Die Bildung und Ausbildung der industriellen Klassen..., S. $24 \mathrm{ff}$. 
vereinzelt die Funktion, den sozialen Status zu bestimmen, sondern sie setzt ihn weitgehend voraus. ${ }^{1}$ Was die Volksschule betrifft, läßt sich die Richtung der politischen Führung zumindest für die Zeit bis 1872 schulpolitisch umschreiben als antiaufklärerisch, antiliberal und klassengebunden. Höhepunkt sind die berüchtigten Stiehlschen Regulative von 1854. Wilhelm I. leitet auch die „Neue Ära” noch ein mit dem Programm, daß Preußen „durch seine Schulen die den verschiedenen Klassen der Bevölkerung nötige Bildung gewähren [soll], ohne diese Klassen über ihre Sphären zu heben."2 Doch auch vor der Revolution von 1848 gab es schon Widerstände gegen eine Erweiterung des Lehrstoffes über den Religionsunterricht hinaus. ${ }^{3}$ Uberhaupt kann man zweifeln, ob die neuhumanistische Schulreform, die auch die alte nachreformatorische Katechismusschule durch neu konzipierte Elementar- und Stadtschulen ablösen wollte, in nennenswertem Umfang realisiert wurde, von fortschrittlichen Kommunen abgesehen. Nach 1819 jedenfalls konnte sich die Reaktion sukzessive der Volksschule bemächtigen oder wenigstens verstärkten Einfluß auf die Volksschulpolitik gewinnen.4

Diese schulpolitische Situation mußte sich auch in der Kanonisierung von Schulbüchern niederschlagen. Rochow hatte, den Bildungs-

1 Vgl. Helmut Schelsky, Schule und Erziehung in der industriellen Gesellschaft, Würzburg 1957, S. 14. - Es entspricht diesem Bild, wenn Pollard die Mobilität für die Frühphase der englischen industriellen Revolution zwar zufallsabhängig (an Protektion gebunden), aber größer veranschlagt als $z u$ einem späteren Zeitpunkt, wo der Weg nach oben zwar stärker rationalisiert und institutionalisiert, das Bildungswesen aber gleichzeitig „Waffe im Kampf um Privilegien” geworden ist. Siehe Die Bildung und Ausbildung der industriellen Klassen..., S. 26.

${ }^{2}$ Siehe Kaiser Wilhelms des Großen Briefe, Reden und Schriften. Ausgewählt und erläutert von Ernst Berner, Band I, 4. Aufl., Berlin 1906, S. 448.

${ }^{3} \mathrm{Vgl}$. Ludwig von der Marwitz, in: Karl Jantke und Dietrich Hilger, Die Eigentumslosen. Der deutsche Pauperismus und die Emanzipationskrise in Darstellungen und Deutungen der zeitgenössischen Literatur, Freiburg/München 1965, S. 140ff.; vgl. Karl Dietrich von Klöden, Jugenderinnerungen, hrsg. und durch einen Umriß seines Weiterlebens vervollständigt von Max Jähns, Leipzig 1874, S. 444.

- Zur preuBischen Volksschulpolitik vgl. Leopold Clausnitzer, Geschichte des preußischen Unterrichtsgesetzes. Mit besonderer Berücksichtigung der Volksschule, 3. Aufl. Berlin 1892; Otto Hattermann, Konservative und liberale Strömungen in der preußischen Volksschulpolitik 1819-1848, Diss. Hamburg 1938; Schulreform in Preußen 1809-1819. Entwürfe und Gutachten. Bearb. von Lothar Schweim [Kleine Pädagogische Texte, Bd 30], Weinheim/Bergstr. 1966; Thomas Nipperdey, Volksschule und Revolution im Vormärz, in: Politische Ideologien und nationalstaatliche Ordnung. Studien zur Geschichte des 19. und 20. Jahrhunderts, Festschrift für Theodor Schieder, München/Wien 1968, S. $117 \mathrm{ff}$. 
notstand der ländlichen Bevölkerung vor Augen, sich an die Abfassung seines Kinderfreundes gemacht und war dabei auf das Wohlwollen des Freiherrn von Zedlitz gestoßen, ebenso wie Ludwig, der von Rochow für die auf Kosten des Armen-Direktoriums in Berlin errichtete Normal-Schule empfohlen worden war und das Interesse der Stadtkinder befriedigen wollte. ${ }^{1}$ Der Durchbruch gegenüber einer rein religiösen Erziehung war bei allem platten Nützlichkeitsdenken unverkennbar. ${ }^{2}$ Die 40er Jahre kennen bereits, im Zuge der Durchsetzung eines effizienten staatlichen Schulwesens verständlich, die Reglementierung von Lehrplänen und Schulbüchern. Mit Otto Schulz, dem Verfasser des Berlinischen Lesebuchs, greift ein Vertreter der Schulverwaltung selbst zur Feder. Wenn man bedenkt, daß Schulz ein Gegner Diesterwegs und verantwortlich für dessen vorzeitige Pensionierung war, kann man sich vorstellen, daß schon in dieser Zeit der Geist der Regulative von 1854 tonangebend war, dem auch der Preußische Kinderfreund zuzurechnen ist. ${ }^{3}$ Es dürfte schwerfallen, hier noch von einer Entfaltung der Persönlichkeit zu sprechen, wie sie das neuhumanistische Bildungsideal anstrebte. Die Stabilisierung des Status quo, die Erhaltung der Klassengesellschaft und die Ausrichtung auf die klassenspezifischen Verhaltensweisen scheinen Inhalt sowohl der Lesebücher wie auch des ihnen zugrundeliegenden Bildungssystems gewesen zu sein.

Eine Verifizierung, Differenzierung oder Korrektur dieses Bildes vom Zusammenhang zwischen Schulbildung und Industrialisierung im 19. Jahrhundert erfordert freilich umfassendere Ansätze, als sie eine Schulbuchanalyse bieten kann. ${ }^{4}$ Innerhalb des Bereichs der Schulbuchanalyse bliebe jedoch noch von Interesse, die Analyse der Volksschullesebücher einer solchen von höheren Schulen gegenüberzustellen. Dabei steht kaum zu erwarten, daß sich hier eine Wirtschaftsbezogenheit der Lesebücher im Sinne der Einübung in den Beruf des Erwerbs als einer klassenspezifischen Verhaltensweise finden wird. Vielmehr deuten alle Anzeichen ${ }^{5}$ darauf hin, wie auch jene Geschich te vom Schatz-

1 Siehe Ludwig, Der Bürgerfreund..., Vorrede.

2 Zur Ökonomisierung des Lehrstoffes seit der Aufklärung bis zum neuhumanistischen Gegenschlag vgl. Fritz Neukamm, Wirtschaft und Schule in Württemberg von 1700-1836, Heidelberg 1956, und das Geleitwort von Eduard Spranger dazu.

agl. Bünger, Entwicklingsgeschichte des Volksschullesebuchs, S. 341ff., S. 351 ; vgl. Antz, Das Lesebuch, S. 207.

4 Vgl. S. 87, Anm. 1.

5 Vgl. Roeder, Geschichte und Kritik des Lesebuchs...; Hans-Georg Herrlitz, Der Lektüre-Kanon des Deutschunterrichts im Gymnasium, Heidelberg 1964; Walther Killy, Zur Geschichte des deutschen Lesebuchs, in: Germanistik eine deutsche Wissenschaft [edition Suhrkamp, 204], Frankfurt 1967, S. 43ff. 
meister und ehemaligen Hirten bei Wackernagel schon gezeigt hat, ${ }^{1}$ $\mathrm{da} B$ die Innerlichkeit des Bildungsbürgers der Wirtschaftswelt entgegengesetzt wird; eine Innerlichkeit, die nun umso leichter zu gewinnen war für eine christlich-konservative, nationalistische oder rassistische Ersatzbefriedigung, wofür Weymars Bericht über den Geist des Geschichtsunterrichts der höheren Schulen im 19. Jahrhundert ein erschütterndes Zeugnis ablegt. ${ }^{2} \mathrm{DaB}$ derartige Ideologien für das Erwerbsstreben keine Konkurrenz bedeuten, zeigt ihre Verwendbarkeit zur erneuten Disziplinierung und Formierung „Unzufriedener”.

Vielleicht haben Schulbücher einen so geringen Wirkungsgrad, daß unsere Schlußfolgerungen mit Recht als übertrieben angesehen werden können. Sie enthüllen aber Ideologie und Intentionen ihrer Verfasser und Herausgeber, stellen zumindest eine nicht unbeachtliche Quelle zur politischen Geistesgeschichte dar. ${ }^{3}$ Wieweit sie die soziale Wirklichkeit beeinflußt haben, läßt sich noch weniger entscheiden als die Frage, ob und wie sie diese Wirklichkeit widerspiegeln, solange nicht empirische Forschung nach der tatsächlichen Stellung des Schulabsolventen in Wirtschaft und Gesellschaft fragt.

1 Siehe S. 115, Anm. 3.

2 Ernst Weymar, Das Selbstverständnis der Deutschen. Ein Bericht über den Geist des Geschichtsunterrichts der höheren Schulen im 19. Jahrhundert, Stuttgart 1961 .

${ }^{3}$ Vgl. das Vorwort von Karl Dietrich Erdmann zu Weymars Selbstverständnis der Deutschen, S. 7. 